\title{
TWISTED ALEXANDER POLYNOMIALS AND SYMPLECTIC STRUCTURES
}

\author{
STEFAN FRIEDL AND STEFANO VIDUSSI \\ Dedicated to the memory of Jerry Levine
}

\begin{abstract}
Let $N$ be a closed, oriented 3-manifold. A folklore conjecture states that $S^{1} \times$ $N$ admits a symplectic structure only if $N$ admits a fibration over the circle. The purpose of this paper is to provide evidence to this conjecture studying suitable twisted Alexander polynomials of $N$, and showing that their behavior is the same as of those of fibered $3-$ manifolds. In particular, we will obtain new obstructions to the existence of symplectic structures and to the existence of symplectic forms representing certain cohomology classes of $S^{1} \times N$. As an application of these results we will show that $S^{1} \times N(P)$ does not admit a symplectic structure, where $N(P)$ is the 0 -surgery along the pretzel knot $P=(5,-3,5)$, answering a question of Peter Kronheimer.
\end{abstract}

\section{INTRODUCTION}

Let $N$ be a $3-$ manifold. Throughout the paper, unless otherwise stated, we will assume that all 3-manifolds are closed, oriented and connected. A 4-manifold is called symplectic if it admits a closed, non-degenerate 2 -form $\omega$ (cf. GS99]).

Thurston [Th76] showed that if $N$ admits a fibration over $S^{1}$, then $S^{1} \times N$ admits a symplectic structure. It is natural to ask whether the converse of this statement holds true; interest on this question was motivated by Taubes' observations on this problem, combined with his results on the Seiberg-Witten invariants of symplectic 4-manifolds (see [Ta98]). We can state this problem in the following form:

Conjecture 1.1. Let $N$ be a 3-manifold. If $S^{1} \times N$ is symplectic, then there exists $\phi \in$ $H^{1}(N)$ such that $(N, \phi)$ fibers over $S^{1}$.

Here we say that $(N, \phi)$ fibers over $S^{1}$ if the homotopy class of maps $N \rightarrow S^{1}$ determined by $\phi \in H^{1}(N)=\left[N, S^{1}\right]$ contains a representative that is a fiber bundle over $S^{1}$; in that case, we will also say that $\phi$ is a fibered class. The purpose of this paper is to use twisted Alexander polynomials to investigate Conjecture 1.1.

The strategy of this paper is to show that, if $S^{1} \times N$ is symplectic, then $N$ satisfies properties that hold for fibered 3-manifold, obtaining this way evidence for Conjecture 1.1 and obstructions to the existence of symplectic forms. This approach is made possible by the fact that we can translate Taubes' constraints on the Seiberg-Witten invariants of a symplectic 4-manifold of the form $S^{1} \times N$ (hence of $N$ ) in terms of the properties of

Date: August 16, 2007.

2000 Mathematics Subject Classification. 57R17; 57M27.

S. Vidussi was partially supported by NSF grant \#0629956. 
the Alexander polynomial of $N$, using the relation between Seiberg-Witten and Alexander invariants of a 3-manifold determined by Meng and Taubes in [MeT96]. This strategy was first used by Kronheimer who proved in [Kr99] that if the 0-surgery $N(K)$ along a knot $K \subset S^{3}$ is such that $S^{1} \times N(K)$ is symplectic, then the Alexander polynomial of $K$ is monic and its degree coincides with twice the genus of $K$. These conditions are known to hold for fibered knots. This approach was generalized by the second author in [Vi03] to all irreducible 3-manifold (see Section 2 for details). This paper uses the twisted Alexander polynomial associated to an epimorphism of the fundamental group of a 3-manifold onto a finite group to strengthen these conclusions. This way we obtain further evidence for Conjecture 1.1 and new obstructions to the existence of symplectic forms.

The main result in this sense is Theorem 4.3. For sake of exposition, we quote here a result that is a slightly weaker version of Theorem 4.3;

Proposition. Let $N$ be an irreducible 3 -manifold such that $S^{1} \times N$ admits a symplectic structure. Then there exists a non-trivial $\phi \in H^{1}(N)$ such that for any epimorphism $\alpha: \pi_{1}(N) \rightarrow G$ onto a finite group $G$ the associated 1 -variable twisted Alexander polynomial $\Delta_{N, \phi}^{\alpha} \in \mathbb{Z}\left[t^{ \pm 1}\right]$ is monic and

$$
\operatorname{deg} \Delta_{N, \phi}^{\alpha}=|G|\|\phi\|_{T}+2 \operatorname{div} \phi_{G} .
$$

Here we denote by $\phi_{G}$ the restriction of $\phi: \pi_{1}(N) \rightarrow \mathbb{Z}$ to $\operatorname{Ker}\left\{\alpha: \pi_{1}(N) \rightarrow G\right\}$, div $\phi_{G} \in \mathbb{N}$ denotes its divisibility and $\|\phi\|_{T}$ is the Thurston norm of $\phi$.

In fact for any $3-$ manifold we have $\operatorname{deg} \Delta_{N, \phi}^{\alpha} \leq|G|\|\phi\|_{T}+2 \operatorname{div} \phi_{G}$. This inequality generalizes McMullen's inequality for the ordinary Alexander polynomial (Lemma 3.9). Note that whenever $\phi$ is a fibered class the equality holds and $\Delta_{N, \phi}^{\alpha}$ is monic (Theorem 4.1). In this sense, this proposition supports Conjecture 1.1.

In practical terms, the previous proposition can be used to show that certain manifolds of the form $S^{1} \times N$, where $N$ is a non-fibered 3-manifold for which the ordinary Alexander polynomial does not provide an obstruction, do not admit any symplectic form. An interesting example in this sense is the manifold $N(P)$ given by 0 -surgery of $S^{3}$ along the pretzel knot $P=(5,-3,5)$. This knot (and so $N(P)$ ) is not fibered, has genus 1 , and its Alexander polynomial equals $\Delta=t^{2}-3 t+1$, so Kronheimer's results do not exclude that $S^{1} \times N(P)$ is symplectic. Kronheimer asked in [Kr99] to determine whether $S^{1} \times N(P)$ is symplectic or not. We have the following.

Proposition. Let $P$ be the pretzel knot $(5,-3,5)$, and let $N(P)$ be the 0 -surgery of $S^{3}$ along $P$. The manifold $S^{1} \times N(P)$ does not admit a symplectic structure.

The proof follows by applying the previous proposition for a suitable choice of the epimorphism $\alpha$. Several other examples, similar to this, are presented. In particular we completely determine for which knots with up to 12 crossings the manifold $S^{1} \times N(K)$ admits a symplectic structure. For many of these knots the ordinary Alexander polynomial does not contain sufficient information. This leads to verifying the following.

Proposition. Conjecture 1.1] holds true for a 3-manifold obtained by 0-surgery along a knot with up to 12 crossings. 
For fibered manifolds $N$ with $b_{1}(N)>1$ the first proposition can be seen as determining new constraints on the size of the symplectic cone of $S^{1} \times N$, as the Künneth component in $H^{1}(N)$ of an integral symplectic form must satisfy the conclusion of the theorem.

In view of the strength of the constraints on fiberability given by the twisted Alexander polynomials, we will present (perhaps optimistically) a conjecture (Conjecture 4.2), stating that the constraints associated to all epimorphisms of the fundamental group of a 3-manifold onto finite groups determine whether the manifold is fibered. We will prove that this conjecture implies Conjecture 1.1, and discuss some arguments in favor of the conjecture.

We finish this introduction with two remarks. First, in this paper we have followed the approach of formulating definitions and results in terms of (twisted) Alexander polynomials and the corresponding Alexander norms. This is convenient because of their wide use, but we would like to point out that the most natural approach would be to use instead the corresponding Reidemeister torsion. Several results (particularly for the $b_{1}(N)=1$ case) have a more elegant presentation when formulated in terms of torsion. Second, some of the results concerning the fiberability of a 3-manifold that are presented here appear (although in different form or generality) in two papers of the first author and Taehee Kim (see [FK05] and [FK06]) as well as, for the case of knots, in previous work of Cha (see [Ch03]) and Goda, Kitano and Morifuji (see GKM05]). However, we have decided for their inclusion both in light of Conjectures 1.1 and 4.2 (as they specify the actual evidence to it) and because the proofs presented are different in nature and have often emerged independently. In particular, the approach presented here is naturally influenced by the viewpoint of Seiberg-Witten theory, and we would like to point out that all our constraints on fiberability (and the McMullen-type inequality) can be obtained with the use of Seiberg-Witten theory alone. In this sense we hope to have progressed (although very modestly) in the direction of better understanding the "intriguing circle of ideas" referred to in the introduction of [MT99].

Organization of the paper: Section 2 summarizes what is known on the relation between Conjecture 1.1 and the ordinary Alexander polynomial. In Section 3 we define the twisted Alexander polynomials and discuss some of their properties, with particular attention to the case of polynomials associated with epimorphisms onto finite groups. In Section 4 we determine the new obstructions to the existence of symplectic structures in terms of twisted Alexander polynomials, and we apply this result to study a family of examples in Section 5. In Section 6 the obstructions are reformulated in terms of certain twisted Alexander polynomials with coefficients in a finite field, which are usually easier to compute. This allows us, in Section 7, to decide Kronheimer's example and determine in the affirmative Conjecture 1.1 for knots with up to 12 crossings. Sections 8 contains some remarks on our conjecture.

Notations and conventions: We assume that all manifolds are closed, oriented and connected. All homology groups and cohomology groups are with respect to $\mathbb{Z}$-coefficients, unless specifically said otherwise. For a knot $K$ in $S^{3}$ we denote the result of zero framed surgery along $K$ by $N(K)$. For a submanifold $X$ of a manifold $M$ we write $\nu X$ for an open tubular neighborhood of $X$ in $M$. Given a prime $p$ the finite field with $p$ elements is denoted by $\mathbb{F}_{p}$. For a 3 -manifold $N$ we write $H=H_{1}(N) / \operatorname{Tor}\left(H_{1}(N)\right)$, i.e. $H$ is the maximal free abelian quotient of $H_{1}(N)$. Given a free abelian group $F$ (by which we always 
mean a finitely generated free abelian group) we use the canonical isomorphisms to identify $H^{1}(N ; F)=\operatorname{Hom}\left(\pi_{1}(N), F\right)=\operatorname{Hom}\left(H_{1}(N), F\right)=\operatorname{Hom}(H, F)$. Given a domain $R$ we denote its quotient field by $Q(R)$. Given a (co)homology class $a$ on $N$ we denote its Poincaré dual by $P D_{N} a$.

Acknowledgment: The authors would like to thank John Hempel, Taehee Kim and Jerry Levine for helpful discussions.

\section{Symplectic Products And the ordinary Alexander Polynomial}

Our first goal is to discuss some properties satisfied by fibered 3-manifolds. In order to present these properties, we will begin by reviewing some definitions that are relevant in what follows.

First, the Thurston (semi)norm (cf. [Th86]): Let $\phi \in H^{1}(N)$. The Thurston norm of $\phi$ is defined as

$$
\|\phi\|_{T}=\min \{-\chi(\hat{S}) \mid S \subset M \text { properly embedded surface dual to } \phi\}
$$

where $\hat{S}$ denotes the result of discarding all connected components of $S$ with positive Euler characteristic. Thurston showed that we can uniquely extend the definition to a continuous norm on $H^{1}(N ; \mathbb{R})$ by requiring linearity. We denote the norm ball $\left\{\phi \in H^{1}(N ; \mathbb{R}) \mid\|\phi\|_{T} \leq\right.$ 1\} by $B_{T}$.

A specific case has particular relevance for us: Given an oriented non-trivial knot $K \subset S^{3}$, let $\phi \in H^{1}(N(K)) \cong \mathbb{Z}$ be a generator. By a result of Gabai Ga87, Theorem 8.8] we have $\|\phi\|_{T}=2 g(K)-2$. Here $g(K)$ is the usual genus of the knot $K$, defined as the minimal genus of a surface bounding $K$.

Second, the Alexander polynomial and the Alexander (semi)norm: We can associate to $N$ the multivariable polynomial $\Delta_{N} \in \mathbb{Z}[H]$, where $H=H_{1}(N) / \operatorname{Tor}\left(H_{1}(N)\right)$, which we can assume, by [Tu86, p. 176], to be symmetric (cf. Section 3.1 for details). We can therefore write $\Delta_{N}=\sum_{g \in H} a_{g} g$, where $a_{-g}=a_{g}$. The Alexander norm of $\phi \in H^{1}(N ; \mathbb{R})$ is then defined as

$$
\|\phi\|_{A}:=2 \max \left\{\phi(g) \mid g \in \operatorname{supp} \Delta_{N}\right\} .
$$

Note that when $\Delta_{N}=0$ this is understood to mean that $\|\phi\|_{A}=0$. The norm ball $B_{A}$ is a convex (possibly non-compact) polyhedron, dual to the Newton polyhedron of $\Delta_{N}$ (cf. McM02 for details). Whenever useful, we will specify in the notation the manifold to which the Alexander and the Thurston norms refers to.

In McM02 McMullen has shown that the Alexander norm of a 3 -manifold $N$ provides a bound by below to the Thurston norm of a cohomology class $\phi \in H^{1}(N)$. McMullen's inequality has the form

$$
\|\phi\|_{A} \leq\|\phi\|_{T}+ \begin{cases}0 & \text { if } b_{1}(N)>1, \\ 2 \operatorname{div} \phi & \text { if } b_{1}(N)=1 .\end{cases}
$$

When the class $\phi$ represents a fibration, this bound becomes an equality, and $\Delta_{N}$ has peculiar properties, as shown from the following theorem, implicitly or explicitly proven, with different techniques, in [Du01], [McM02], [Vi03], and [FK06]. Remember that Thurston 
[Th86] showed that if a class $\phi \in H^{1}(N)$ is fibered then it lies in the cone over some topdimensional face $F_{T}$. (Here and in what follows we will always consider open faces for polyhedra.) Furthermore a class $\phi \in H^{1}(N)$ is fibered if and only if all the lattice points in the cone $\mathbb{R}_{+} F_{T}$ over the same top-dimensional face $F_{T}$ of the Thurston unit ball are fibered. The case of completely degenerate norm is included in this and later statements by interpreting $F_{T}$ as the unique "face at infinity", dual to the trivial element of $H$, so that $\mathbb{R}_{+} F_{T}=H^{1}(N ; \mathbb{R}) \backslash\{0\}$. Note that in the case $b_{1}(N)=1$ the (top-dimensional) faces are two discrete points, in particular they are open. Denote by $e_{F_{T}} \in H$ the (Poincaré dual of the) Euler class of the fibrations in $F_{T}$. (Strictly speaking, this is the image of the Euler class under the quotient map $H_{1}(N) \rightarrow H$.)

Theorem 2.1. Let $N \neq S^{1} \times S^{2}$ be a fibered 3 -manifold, and let $F_{T}$ be a fibered face of the Thurston unit ball $B_{T}$. Then the following holds true:

(1) For all $\phi \in \mathbb{R}_{+} F_{T} \cap H^{1}(N)$

$$
\|\phi\|_{A}=2 \phi(\lambda)=\|\phi\|_{T}+ \begin{cases}0 & \text { if } b_{1}(N)>1, \\ 2 \operatorname{div} \phi & \text { if } b_{1}(N)=1,\end{cases}
$$

where $\lambda \in H$ is a vertex of the Newton polyhedron of $\Delta_{N}$ with coefficient \pm 1 . If $b_{1}(N)>1$, then $\lambda=-\frac{1}{2} e_{F_{T}}$ and $F_{T} \subset F_{A}$ (where $F_{A}$ is the top-dimensional face of the Alexander unit ball dual to $\lambda$ ) and if $b_{1}(N)=1$, then $\lambda=-\frac{1}{2} e_{F_{T}}+1$.

(2) For all $\phi \in \mathbb{R}_{+} F_{T} \cap H^{1}(N), \Delta_{N, \phi}$ is monic and $\operatorname{deg} \Delta_{N, \phi}=\|\phi\|_{T}+2 \operatorname{div} \phi$.

Here $\Delta_{N, \phi}$ is the 1-variable Alexander polynomial associated to $\phi$, viewed as element of $\operatorname{Hom}(H, \mathbb{Z})$ (cf. Section 3.1 for details). Furthermore note that in the case $b_{1}(N)=1$ the choice of a face $F_{T}$ corresponds to the choice of an isomorphism $H \rightarrow \mathbb{Z}$, i.e. we can identify $H$ and $\mathbb{Z}$. The last equation of part (1) in the preceding theorem has to be understood using this identification.

These properties (that generalize classical results of Neuwirth for knots) are short of characterizing fibered classes, but provide useful and often effective constraints for fiberability.

Let us now assume that $S^{1} \times N$ admits a symplectic form $\omega$, whose cohomology class with no loss of generality can be taken in the integer lattice of $H^{2}\left(S^{1} \times N ; \mathbb{R}\right)$. Decompose $[\omega]$ according to its Künneth decomposition, and let $\varphi$ be its Künneth component in $H^{1}(N)$, normalized so that $[\omega]=[d t] \wedge \varphi+\eta$, where $[d t]$ is the generator of $H^{1}\left(S^{1}\right)$ and $\eta \in H^{2}(N)$. Denote by $K \in H^{2}\left(S^{1} \times N\right)$ the canonical class of the symplectic structure: such class is well-known to be the pull-back of a class in $H^{2}(N)$ that we denote with the same symbol. Evidence to Conjecture 1.1 comes from the fact that such a manifold (under the additional hypothesis of irreducibility) satisfies the same properties of Theorem 2.1, as shown in [Vi03] (and [Vi99] for $b_{1}(N)=1$ ):

Theorem 2.2. Let $N$ be an irreducible 3-manifold such that $S^{1} \times N$ admits an integral symplectic structure with Künneth component $\varphi \in H^{1}(N)$. Then there exists a top-dimensional face $F_{T}$ of the Thurston unit ball with $\varphi \in \mathbb{R}_{+} F_{T}$ for which the same properties as in (1) and (2) of Theorem 2.1 hold true, with $\lambda$ equal to $\frac{1}{2} P D_{N} K$ if $b_{1}(N)>1$ and $\frac{1}{2} P D_{N} K+1$ if $b_{1}(N)=1$. 
For future reference and completeness, we summarize the idea of the proof of [Vi03]. This proof combines (generalizing ideas of Kronheimer, see [Kr98] and [Kr99]) the following ingredients, connected by the relation between the Seiberg-Witten theory of $S^{1} \times N$ and the Alexander and Thurston norm of $N$. First, Taubes' results on symplectic 4-manifolds impose conditions on the convex hull of basic classes of Seiberg-Witten theory, in particular on $K$, and its relation with $\varphi$. In particular, $\varphi$ has maximal pairing, among all elements of $\operatorname{supp} \Delta_{N}$, with $\lambda$. This implies that $\varphi \in \mathbb{R}_{+} F_{A}$, where $F_{A}$ is the face dual to $\lambda$. Second, Donaldson's Theorem guarantees the existence of a symplectic representative for the class Poincaré dual to (a sufficiently high multiple of) $\omega$. Last, we apply to such a representative Kronheimer's refined adjunction inequality for manifolds of the form $S^{1} \times N$. These constraints prove the equalities of Theorem 2.2 for the norms of $\varphi$. Using the openness of the symplectic condition we can extend this result, when $b_{1}(N) \geq 2$, to some open cone in $H^{1}(N ; \mathbb{R})$, determined by symplectic forms all having canonical class $K$. This cone, where the norms coincide, must be contained in $\mathbb{R}_{+} F_{A}$. This implies that $\varphi \in \mathbb{R}_{+} F_{T}$, where $F_{T}$ is some (open) top-dimensional face of $B_{T}$. The relation $F_{T} \subset F_{A}$ is then a consequence of McMullen's inequality.

\section{Twisted Alexander polynomials and finite covers}

3.1. Twisted Alexander modules and their polynomials. In this section we are going to define (twisted) Alexander polynomials. These were introduced, for the case of knots, by Xiao-Song Lin in 1990 (published in [L01]), and his definition was later generalized to 3-manifolds by Jiang and Wang [JW93, Wada [Wa94, Kirk-Livingston [KL99] and Cha Ch03. Our definition below is a generalization of Cha's approach.

For the remainder of this section let $F$ be a free abelian group and let $R$ be $\mathbb{Z}$ or the field $\mathbb{F}_{p}:=\mathbb{Z} / p \mathbb{Z}$ where $p$ is a prime number. Let $N$ be a compact manifold. Let $\phi \in$ $\operatorname{Hom}(H, F)$ be a non-trivial homomorphism. Through the homomorphism $\phi, \pi_{1}(N)$ acts on $F$ by translations. Let now $A$ be a free finite $R-$ module and $\alpha: \pi_{1}(N) \rightarrow \operatorname{Aut}_{R}(A)$ be a representation. We write $A[F]=A \otimes_{R} R[F]$. We get a representation

$$
\begin{aligned}
\alpha \otimes \phi: \quad \pi_{1}(N) & \rightarrow \operatorname{Aut}(A[F]) \\
g & \mapsto\left(\sum_{i} a_{i} \otimes f_{i} \mapsto \sum_{i} \alpha(g)\left(a_{i}\right) \otimes\left(f_{i}+\phi(g)\right) .\right.
\end{aligned}
$$

We can therefore view $A[F]$ as a left $\mathbb{Z}\left[\pi_{1}(N)\right]$-module. Note that this module structure commutes with the natural right $R[F]-$ multiplication on $A[F]$.

Let $\tilde{N}$ be the universal cover of $N$. Note that $\pi_{1}(N)$ acts on the left on $\tilde{N}$ as group of deck transformation. The chain groups $C_{*}(\tilde{N})$ are in a natural way right $\mathbb{Z}\left[\pi_{1}(N)\right]$ modules, with the right action on $C_{*}(\tilde{N})$ defined via $\sigma \cdot g:=g^{-1} \sigma$, for $\sigma \in C_{*}(\tilde{N})$. We can form by tensor product the chain complex $C_{*}(\tilde{N}) \otimes_{\mathbb{Z}\left[\pi_{1}(N)\right]} A[F]$. Now define $H_{*}(N ; A[F]):=$ $H_{*}\left(C_{*}(\tilde{N}) \otimes_{\mathbb{Z}\left[\pi_{1}(N)\right]} A[F]\right)$, which inherit the structure of right $R[F]$-modules. These modules take the name of twisted Alexander modules.

Our next goal is to define invariants out of these modules. The $R[F]$-modules $H_{i}(N ; A[F])$ are finitely presented and finitely related $R[F]$-modules since $R[F]$ is Noetherian. Therefore $H_{i}(N ; A[F])$ has a free $R[F]$-resolution

$$
R[F]^{r} \stackrel{S_{i}}{\rightarrow} R[F]^{s} \rightarrow H_{i}(N ; A[F]) \rightarrow 0
$$


of finite $R[F]$-modules, where we can always assume that $r \geq s$.

Definition 3.1. The $i$-th twisted Alexander polynomial of $(N, \alpha, \phi)$ is defined to be the order of the $R[F]$-module $H_{i}(N ; A[F])$, i.e. the greatest common divisor of the $s \times s$ minors of the $s \times r$-matrix $S_{i}$. It is denoted by $\Delta_{N, \phi, i}^{\alpha} \in R[F]$.

Note that this definition only makes sense since $R[F]$ is a UFD. It is well-known that $\Delta_{N, \phi, i}^{\alpha}$ is well-defined only up to multiplication by a unit in $R[F]$ and its definition is independent of the choice of the resolution. Whenever we drop $i$ from the notation, we will refer to the first Alexander polynomial. If it is clear which manifold $N$ we are referring to, we will drop it from the notation.

In this paper we will be mostly interested in the case where $F=\mathbb{Z}=\langle t\rangle$ and $\phi$ is identified with an element of $H^{1}(N)$ (so that $\Delta_{N, \phi, i} \in R\left[t^{ \pm 1}\right]$ ) and in the case where $\phi$ is the identity map of $H$. In the latter case we will simply write $\Delta_{N, i}^{\alpha}$. Also, we will write $\Delta_{N, \phi, i}$ in the case that $\alpha: \pi_{1}(N) \rightarrow \mathrm{GL}(\mathbb{Z}, 1)$ is the trivial representation. According to these conventions, when $R=\mathbb{Z}, \Delta_{N} \in \mathbb{Z}[H]$ is the ordinary multivariable Alexander polynomial of $N$.

Note that $\Delta_{S^{3} \backslash \nu K} \in \mathbb{Z}\left[t^{ \pm 1}\right]$ is just the classical Alexander polynomial $\Delta_{K}$ of a knot. Furthermore recall that $H_{1}\left(S^{3} \backslash \nu K ; \mathbb{Z}\left[t^{ \pm 1}\right]\right) \cong H_{1}\left(N(K) ; \mathbb{Z}\left[t^{ \pm 1}\right]\right)$. In particular $\Delta_{N(K)}=$ $\Delta_{S^{3} \backslash \nu K}=\Delta_{K}$.

3.2. Twisted Alexander polynomials and finite covers. For the most part of this paper we will be concerned with a particular type of representation: Let $\alpha: \pi_{1}(N) \rightarrow G$ be an epimorphism onto a finite group $G$. Then we get an induced representation $\pi_{1}(N) \rightarrow$ $\operatorname{Aut}_{R}(R[G])$ given by left multiplication. We denote this representation by $\alpha$ as well.

To study this case, we first need some definitions and results around the map $\alpha$. Denote the normal $G$-cover of $N$ induced by $\alpha: \pi_{1}(N) \rightarrow G$ by $N_{G}$ (a compact manifold), with $\pi: N_{G} \rightarrow N$ the covering map. We have an exact sequence

$$
1 \rightarrow \pi_{1}\left(N_{G}\right) \stackrel{\pi_{*}}{\rightarrow} \pi_{1}(N) \stackrel{\alpha}{\rightarrow} G \rightarrow 1 .
$$

As $H$ is free abelian, the inclusion $\pi_{*}: \pi_{1}\left(N_{G}\right) \rightarrow \pi_{1}(N)$ induces a map $\pi_{*}: H_{G} \rightarrow H$, where $H_{G}$ is the maximal free abelian quotient of $H_{1}\left(N_{G}\right)$. We have therefore a canonical homomorphism $\operatorname{Hom}(H, F) \rightarrow \operatorname{Hom}\left(H_{G}, F\right)$ so that given any homomorphism $\phi: H \rightarrow F$ we can consider the induced homomorphism $\phi_{G}:=\pi^{*} \phi: H_{G} \rightarrow F$.

Lemma 3.2. Let $\alpha: \pi_{1}(N) \rightarrow G$ be an epimorphism to a finite group $G$.

(1) $\pi^{*}: \operatorname{Hom}(H, F) \rightarrow \operatorname{Hom}\left(H_{G}, F\right)$ is injective (in particular $\left.b_{1}\left(N_{G}\right) \geq b_{1}(N)\right)$.

(2) Given $\phi \in \operatorname{Hom}(H, F)$ we have $r k(\operatorname{Im} \phi)=r k\left(\operatorname{Im} \phi_{G}\right)$.

Proof. Recall that we identify $\operatorname{Hom}(H, F)=\operatorname{Hom}\left(\pi_{1}(N), F\right)$ and, $\operatorname{similarly}, \operatorname{Hom}\left(H_{G}, F\right)=$ $\operatorname{Hom}\left(\pi_{1}\left(N_{G}\right), F\right)$. Using our identifications we have $\pi^{*} \phi=\left.\phi\right|_{\pi_{1}\left(N_{G}\right)}$. Let $\phi \in \operatorname{ker} \pi^{*}$. For any $g \in \pi_{1}(N)$ we have $\phi(g)^{|G|}=\phi\left(g^{|G|}\right)=0$ as $g^{|G|} \in \operatorname{ker} \alpha=\pi_{1}\left(N_{G}\right)$. But $\phi^{|G|}=0$ implies $\phi=0$, as $\operatorname{Hom}\left(\pi_{1}(N), F\right)$ is torsion-free. This proves the first statement. The second statement is immediate.

Note that $\operatorname{div} \phi_{G}=\operatorname{div}\left(\phi \circ \pi_{*}\right) \geq \operatorname{div} \phi \cdot \operatorname{div} \pi_{*}$ and equality holds by fiat if $b_{1}(N)=1$. We mention also that $\operatorname{div} \pi_{*}$ divides $|G|$, a result that is useful in interpreting some of the formulae that follow. Proving such relation is a straightforward exercise in group theory based on Lagrange's formula. 
The key tool of this paper is a relation between the twisted Alexander polynomials of $N$ determined by $\alpha$ and (part of) the ordinary Alexander polynomials for the corresponding cover $N_{G}$. This relation follows from an isomorphism of Alexander modules that is a straightforward generalization of the Eckmann-Shapiro Lemma in group cohomology. We have the following:

Lemma 3.3. Let $\phi: H \rightarrow F$ be a non-trivial homomorphism to a free abelian group $F$, then

$$
\Delta_{N, \phi, i}^{\alpha}=\Delta_{N_{G}, \phi_{G}, i} \in R[F] .
$$

Proof. The proof of this statement follows the steps of the proof of the Eckmann-Shapiro Lemma: first observe that $R[F]$ inherits a left $\mathbb{Z}\left[\pi_{1}\left(N_{G}\right)\right]$-module structure by restriction of its $\mathbb{Z}\left[\pi_{1}(N)\right]$-module structure. Secondly note that the universal covers $\tilde{N}$ and $\tilde{N}_{G}$ of $N$ and $N_{G}$ are the same. Using associativity of the tensor product we have the following canonical isomorphisms of chain complexes of $R[F]$-modules

$$
\begin{aligned}
C_{*}\left(\tilde{N}_{G}\right) \otimes_{\mathbb{Z}\left[\pi_{1}\left(N_{G}\right)\right]} R[F] & =C_{*}(\tilde{N}) \otimes_{\mathbb{Z}\left[\pi_{1}\left(N_{G}\right)\right]} R[F] \\
& \cong C_{*}(\tilde{N}) \otimes_{\mathbb{Z}\left[\pi_{1}(N)\right]}\left(\mathbb{Z}\left[\pi_{1}(N)\right] \otimes_{\mathbb{Z}\left[\pi_{1}\left(N_{G}\right)\right]} R[F]\right) \\
& \cong C_{*}(\tilde{N}) \otimes_{\mathbb{Z}\left[\pi_{1}(N)\right]} R[G][F],
\end{aligned}
$$

where to establish the last equality we have used the fact that $\mathbb{Z}\left[\pi_{1}(N)\right] \otimes_{\mathbb{Z}\left[\pi_{1}\left(N_{G}\right)\right]} R[F]$ is canonically isomorphic to $R[G][F]$. This proves the lemma, since $\Delta_{N_{G}, \phi_{G}, i}$ is defined using the first chain complex and $\Delta_{N, \phi, i}^{\alpha}$ is defined using the last chain complex.

As in the ordinary case, the twisted $i$-th Alexander polynomials for $i=0,2,3$ contain only a modest amount of information.

Lemma 3.4. Let $N$ be a 3-manifold, $\phi \in \operatorname{Hom}(H, F)$ a non-trivial homomorphism to a free abelian group and $\alpha: \pi_{1}(N) \rightarrow G$ an epimorphism to a finite group $G$ such that $\Delta_{N, \phi}^{\alpha} \neq 0$. Then $\Delta_{N, \phi, 3}^{\alpha}=1$ and

$$
\Delta_{N, \phi, 0}^{\alpha}=\Delta_{N, \phi, 2}^{\alpha}= \begin{cases}1 & \text { if } \operatorname{rk}\left(\operatorname{Im} \phi_{G}\right)>1, \\ \left(t^{\text {div } \phi_{G}}-1\right) & \text { if } \operatorname{Im} \phi_{G}=\left\langle t^{d i v \phi_{G}}\right\rangle, t \in F \text { indivisible. }\end{cases}
$$

Proof. The calculation follows from Lemma 3.3 and the corresponding well-known result for the ordinary Alexander polynomial.

A priori $\Delta_{N, \phi}^{\alpha}$ is defined only up to units in $R[F]$. Using Lemma 3.3 and a result of Turaev on the first Alexander polynomial of closed 3-manifolds ([Tu86, p. 176]) we can actually suppress the ambiguity of the first twisted Alexander polynomial relative to multiplication by an element of $F$. Therefore we will be able to assume, whenever suitable, that $\Delta_{N, \phi}^{\alpha} \in R[F]$ is symmetric and well-defined up to a unit of $R$.

Consider now the complex $C_{*}(N ; R[G][F])=C_{*}(\tilde{N}) \otimes_{\mathbb{Z}\left[\pi_{1}(N)\right]} R[G][F]$. Equip $N$ with a finite CW-structure. Then picking lifts of ordered cells from $N$ to $\tilde{N}$ and using the canonical basis of $R[G]$ as an $R$-module we can view $C_{*}(N ; R[G][F])$ as a based complex of $R[F]$-modules. Let $Q(F)$ denote the field of fractions of $R[F]$. It follows from Lemma 3.4 and the fact that $Q(F)$ is flat over $R[F]$ that $C_{*}(N ; R[G][F]) \otimes_{R[F]} Q(F)$ is acyclic if and only if $\Delta_{N, \phi}^{\alpha} \neq 0$. When this complex is acyclic we can consider its Reidemeister-Franz 
torsion $\tau_{N, \phi}^{\alpha} \in(Q(F) \backslash\{0\}) /\{ \pm r f \mid f \in F, r$ unit in $R\}$. Otherwise we set $\tau_{N, \phi}^{\alpha}=0$. It follows from [Tu01, Theorem 4.7] together with Lemma 3.4 that $\tau_{N, \phi}^{\alpha} \neq 0$ if and only if $\Delta_{N, \phi}^{\alpha} \neq 0$, furthermore if $\Delta_{N, \phi}^{\alpha} \neq 0$, then

$$
\tau_{N, \phi}^{\alpha}=\prod_{i=0}^{3}\left(\Delta_{N, \phi, i}^{\alpha}\right)^{(-1)^{i+1}} \in Q(F) /\{ \pm r f \mid f \in F, r \text { unit in } R\} .
$$

We denote by $\tau_{N}^{\alpha}$ the torsion associated to the identity map of $H$, which corresponds to the multivariable Alexander polynomial (e.g. $\tau_{N}$ is Milnor's torsion). One of the properties of torsion is its functoriality, encoded in the following theorem:

Theorem 3.5. Let $\rho: R \rightarrow \tilde{R}$ be a ring homomorphism and let $\phi$ be a non-trivial element of $\operatorname{Hom}(H, F)$. These induce a ring homomorphism $\rho \otimes \phi: R[H] \rightarrow \tilde{R}[F]$. Now let

$$
Q=\left\{f g^{-1} \mid f, g \in R[H],(\rho \otimes \phi)(g) \neq 0\right\} \subset Q(R[H]) .
$$

Then $\tau_{N, \phi}^{\alpha} \in Q$. Furthermore $\rho \otimes \phi$ extends to a homomorphism $Q \rightarrow Q(\tilde{R}[F])$ and

$$
(\rho \otimes \phi)\left(\tau_{N}^{\alpha}\right)=\tau_{N, \phi}^{\alpha}
$$

this equality taking value in $\tilde{R}[F]$ for $b_{1}(N) \geq 2$ and in $Q(\tilde{R}[F])$ for $b_{1}(N)=1$.

Proof. The proof follows again from Lemma 3.3 and functoriality for the ordinary torsion, proved in [Tu86, Theorem 1.1.3]. (Note that such result is stated therein only for $R=\tilde{R}=$ $\mathbb{Z}$, but the proof carries over to the general case.)

The following is an immediate corollary to Lemma 3.4 and Theorem 3.5 .

Proposition 3.6. Let $N$ be a 3-manifold and let $\alpha: \pi_{1}(N) \rightarrow G$ be an epimorphism onto a finite group. Let $\pi_{*}: H_{G} \rightarrow H$ be the induced map. Then the twisted Alexander polynomials of $N$ and the ordinary Alexander polynomial of $N_{G}$ satisfy the following relations:

If $b_{1}\left(N_{G}\right)>1$, then

$$
\Delta_{N}^{\alpha}= \begin{cases}\pi_{*}\left(\Delta_{N_{G}}\right) & \text { if } b_{1}(N)>1, \\ \left(t^{d i v \pi_{*}}-1\right)^{2} \pi_{*}\left(\Delta_{N_{G}}\right) & \text { if } b_{1}(N)=1, \text { Im } \pi_{*}=\left\langle t \text { div } \pi_{*}\right\rangle, t \in H \text { indivisible. }\end{cases}
$$

If $b_{1}\left(N_{G}\right)=1$, then $b_{1}(N)=1$ and

$$
\Delta_{N}^{\alpha}=\pi_{*}\left(\Delta_{N_{G}}\right) .
$$

Proof. First assume that $\pi_{*}\left(\Delta_{N_{G}}\right)=0$. Distinguishing the cases where $\Delta_{N_{G}}=0$ and $\Delta_{N_{G}} \neq 0$, and applying in the latter case Lemma 3.4 and Equation (1), this implies that $\pi_{*}\left(\tau_{N_{G}}\right)=0$. It follows from Theorem 3.5, applied to $\pi_{*} \in \operatorname{Hom}\left(H_{G}, H\right)$, that $\tau_{N_{G}, \pi_{*}}=0$, hence $\Delta_{N_{G}, \pi_{*}}=\Delta_{N}^{\alpha}=0$. Now assume that $\pi_{*}\left(\Delta_{N_{G}}\right) \neq 0$; then we have by Theorem 3.5 and Equation (11) that

$$
\pi_{*}\left(\prod_{i=0}^{3}\left(\Delta_{N_{G}, i}\right)^{(-1)^{i+1}}\right)=\prod_{i=0}^{3}\left(\Delta_{N_{G}, \pi_{*}, i}\right)^{(-1)^{i+1}} .
$$

Apply now Lemma 3.3 choosing as $\phi$ the identity map of $H$ (observing that, for this choice, $\phi_{G}=\pi_{*}$ ) and apply Lemma 3.4 twice. Using Lemma 3.2, which implies that $b_{1}(N)=$ $\operatorname{rk}\left(\operatorname{Im} \pi_{*}\right)$, Equations (2) and (3) follow. 
The proposition above asserts that the twisted Alexander polynomial of $N$ is determined by the Alexander polynomial of $N_{G}$ or, stated otherwise, by the Seiberg-Witten invariants of $N_{G}$.

3.3. Twisted Alexander norms and Thurston norm. We can introduce a norm on $H^{1}(N ; \mathbb{R})$ determined by the (symmetric) twisted Alexander polynomial $\Delta_{N}^{\alpha}$, by mimicking McMullen's definition of the Alexander norm. Precisely we have the following:

Definition 3.7. Let $\Delta_{N}^{\alpha}$ be the twisted Alexander polynomial associated to an epimorphism $\alpha: \pi_{1}(N) \rightarrow G$. The twisted Alexander norm associated to $\alpha$ is the (semi)norm on $H^{1}(N ; \mathbb{R})$ defined as

$$
\|\phi\|_{A}^{\alpha}:=2 \max \left\{\phi(g) \mid g \in \operatorname{supp} \Delta_{N}^{\alpha} \subset H\right\} .
$$

As for the ordinary case, this is understood to mean that $\|\phi\|_{A}^{\alpha}=0$ if $\Delta_{N}^{\alpha}=0$.

This norm satisfies, from its definition, all the usual properties of the ordinary Alexander norm. In particular, its unit ball $B_{A}^{\alpha}$ is a convex (possibly non-compact) polyhedron, dual to the Newton polyhedron of $\Delta_{N}^{\alpha}$. Whenever useful, we will specify in the notation the manifold to which the norm refers to.

Let $\phi \in H^{1}(N)$. We can think of $\phi$ as an element of the integer lattice of $H^{1}(N ; \mathbb{R})$. As in the classical Alexander theory, there is a relation between the degree of $\Delta_{N, \phi}^{\alpha}$ and the twisted Alexander norm of $\phi$. (Note that for $f=\sum_{i=n}^{m} a_{i} t^{i}, a_{n} a_{m} \neq 0$ we define $\operatorname{deg}(f)=m-n$, furthermore we write $\operatorname{deg}(0)=-\infty$ with the understanding that $-\infty<a$ for any $a \in \mathbb{Z}$.) The latter, moreover, is related to the ordinary Alexander norm of $\phi_{G}$ on $N_{G}$. The form of these relations is expressed in the following proposition.

Proposition 3.8. Let $\phi$ be a non-trivial element of $H^{1}(N)$; then the following inequalities hold true:

(4) $\operatorname{deg} \Delta_{N, \phi}^{\alpha} \leq\|\phi\|_{A, N}^{\alpha}+\left\{\begin{array}{ll}2 \operatorname{div} \phi_{G} \\ 0 \\ 0\end{array} \leq\left\|\phi_{G}\right\|_{A, N_{G}}+ \begin{cases}2 \operatorname{div} \phi_{G} & \text { if } b_{1}\left(N_{G}\right) \geq b_{1}(N)>1, \\ 2 \operatorname{div} \phi_{G} & \text { if } b_{1}\left(N_{G}\right)>b_{1}(N)=1, \\ 0 & \text { if } b_{1}\left(N_{G}\right)=b_{1}(N)=1 .\end{cases}\right.$

The first relation (provided that $\Delta_{N}^{\alpha} \neq 0$ ) is an equality for all $\phi$ in the cone over the topdimensional faces of the unit ball of the twisted Alexander norm (in particular, whenever $\left.b_{1}(N)=1\right)$.

Proof. Theorem 3.5, applied to $\phi \in \operatorname{Hom}(H, \mathbb{Z})$ asserts that, denoting $\Delta_{N, \phi}^{\alpha}=\sum a_{i} t^{i}$ and $\Delta_{N}^{\alpha}=\sum_{g \in H} b_{g} g$, we have

$$
\sum a_{i} t^{i}= \begin{cases}\left(t^{\operatorname{div} \phi_{G}}-1\right)^{2} \phi\left(\Delta_{N}^{\alpha}\right)=\left(t^{\operatorname{div} \phi_{G}}-1\right)^{2} \sum_{g \in H} b_{g} t^{\phi(g)} & \text { if } b_{1}(N)>1, \\ \phi\left(\Delta_{N}^{\alpha}\right)=\sum_{g \in H} b_{g} t^{\phi(g)} & \text { if } b_{1}(N)=1 .\end{cases}
$$

This implies the first inequality. Whenever $\phi$ is in the cone over a top-dimensional face of the unit ball of the twisted Alexander norm it attains its maximum value at a single $g \in \operatorname{supp} \Delta_{N}^{\alpha} \subset H$ which is dual to that face. In that case, the equality holds. For the second inequality, we can use Proposition 3.6 to determine the relationship between the 
twisted Alexander norm of $N$ and the Alexander norm of $N_{G}$. Write $\Delta_{N_{G}}=\sum_{g \in H_{G}} c_{g} g \in$ $R\left[H_{G}\right]$. Let us consider first the cases where $b_{1}\left(N_{G}\right) \geq b_{1}(N)>1$ or $b_{1}\left(N_{G}\right)=b_{1}(N)=1$ : using Proposition 3.6, $\Delta_{N}^{\alpha}=\sum_{g \in H_{G}} c_{g}\left(\pi_{*} g\right) \in R[H]$; it follows that the support of $\Delta_{N}^{\alpha}$ is contained in the image of the support of $\Delta_{N_{G}}$, and when $b_{1}\left(N_{G}\right)>b_{1}(N)$ this inclusion may happen to be strict. We deduce that, for all $\phi \in H^{1}(N ; \mathbb{R})$,

$$
\|\phi\|_{A, N}^{\alpha}=2 \max \left\{\phi\left(\pi_{*} g\right) \mid \pi_{*} g \in \operatorname{supp} \Delta_{N}^{\alpha}\right\} \leq 2 \max \left\{\phi_{G}(g) \mid g \in \operatorname{supp} \Delta_{N_{G}}\right\}=\left\|\phi_{G}\right\|_{A, N_{G}} .
$$

This gives the first and third line of Equation (4). In the remaining case where $b_{1}\left(N_{G}\right)>$ $b_{1}(N)=1$ Proposition 3.6 gives $\Delta_{N}^{\alpha}=\left(t^{d i v \pi_{*}}-1\right)^{2} \sum_{g \in H_{G}} c_{g}\left(\pi_{*} g\right) \in R[H]$ which implies that, for all $\phi \in H^{1}(N)$,

$$
\begin{aligned}
\|\phi\|_{A, N}^{\alpha} & =2 \operatorname{div} \pi_{*} \cdot \operatorname{div} \phi+2 \max \left\{\phi\left(\pi_{*} g\right) \mid \pi_{*} g \in \operatorname{supp} \Delta_{N}^{\alpha}\right\} \\
& \leq 2 \operatorname{div} \phi_{G}+2 \max \left\{\phi_{G}(g) \mid g \in \operatorname{supp} \Delta_{N_{G}}\right\} \\
& =2 \operatorname{div} \phi_{G}+\left\|\phi_{G}\right\|_{A, N_{G}} .
\end{aligned}
$$

This completes the second line of the inequality and finishes the proof.

We can now examine the relationship between the twisted Alexander norm of $N$ and the Thurston norm, to obtain an inequality à la McMullen. We have the following Lemma:

Lemma 3.9. Let $\phi \in H^{1}(N)$ be non-trivial. Then we have the following inequality:

$$
\|\phi\|_{A}^{\alpha} \leq|G| \cdot\|\phi\|_{T}+ \begin{cases}0 & \text { if } b_{1}(N)>1, \\ 2 \operatorname{div} \phi_{G} & \text { if } b_{1}(N)=1 .\end{cases}
$$

Proof. Corollary 6.13 of [Ga83] states that

$$
|G| \cdot\|\phi\|_{T, N}=\left\|\phi_{G}\right\|_{T, N_{G}}
$$

Combined with McMullen's inequalities for $N_{G}$ and (44) this gives the desired inequalities.

\section{MAin Results}

4.1. Finite covers and fibered manifolds. Let $N$ be a $3-$ manifold and let $\alpha: \pi_{1}(N) \rightarrow G$ be an epimorphism to a finite group $G$ and denote as above by $\pi: N_{G} \rightarrow N$ the induced covering map. Assume that $(N, \phi)$ fibers over $S^{1}$ for $\phi \in H^{1}(N)$ : then it is well known that $\left(N_{G}, \phi_{G}\right)$ fibers over $S^{1}$ too. (In terms of differential forms, the fact that $\phi$ is fibered corresponds to the fact that there exists a non-degenerate closed 1-form $\nu \in \Omega^{1}(N, \mathbb{R})$, unique up to isotopy, representing the corresponding class in $H_{D R}^{1}(N ; \mathbb{R})$. The representative for $\phi_{G}$ is then given by $\pi^{*} \nu$.) The Euler class of the fibration $\phi_{G}$, as element of $H_{G}$, is given by $e_{F_{T, N_{G}}}=P D_{N_{G}}\left(\pi^{*} P D_{N}\left(e_{F_{T, N}}\right)\right)$.

We want to analyze the properties of the twisted Alexander polynomial of a fibered $3-$ manifold. For the rest of the section, we are going to fix $R=\mathbb{Z}$.

We can apply Theorem 2.1 and Proposition 3.6 to $N_{G}$ to get a constraint on the twisted Alexander and Thurston norms of a fibered class, that is a "twisted version" of Theorem 2.1. Precisely, we have the following: 
Theorem 4.1. Let $N \neq S^{1} \times S^{2}$ be a fibered $3-$ manifold, $F_{T}$ be a fibered face of the Thurston unit ball, and let $\alpha: \pi_{1}(N) \rightarrow G$ be an epimorphism onto a finite group. Then the following hold true:

(1) For all $\phi \in \mathbb{R}_{+} F_{T} \cap H^{1}(N)$

$$
\|\phi\|_{A}^{\alpha}=2 \phi\left(\lambda_{G}\right)=|G|\|\phi\|_{T}+ \begin{cases}0 & \text { if } b_{1}(N)>1 \\ 2 \operatorname{div} \phi_{G} & \text { if } b_{1}(N)=1\end{cases}
$$

where $\lambda_{G}$ is a vertex of the Newton polyhedron of $\Delta_{N}^{\alpha}$ with coefficient \pm 1 . If $b_{1}(N)>$ 1 , then $\lambda_{G}=-\frac{|G|}{2} e_{F_{T}}=|G| \lambda$ and $F_{T} \subset|G| F_{A}^{\alpha}$ (where $F_{A}^{\alpha}$ is the top-dimensional face of the Alexander unit ball dual to $\left.\lambda_{G}\right)$; if $b_{1}(N)=1$, then $\lambda_{G}=-\frac{|G|}{2} e_{F_{T}}+$ $\operatorname{div} \pi_{*}=|G| \lambda+\operatorname{div} \pi_{*}-|G|$.

(2) For all $\phi \in \mathbb{R}_{+} F_{T} \cap H^{1}(N), \Delta_{N, \phi}^{\alpha} \in \mathbb{Z}\left[t^{ \pm 1}\right]$ is monic and $\operatorname{deg} \Delta_{N, \phi}^{\alpha}=|G|\|\phi\|_{T}+$ $2 \operatorname{div} \phi_{G}$.

Proof. Write $\Delta_{N_{G}}=\sum_{g \in H_{G}} c_{g} g$. As $\left(N_{G}, \phi_{G}\right)$ fibers, Theorem 2.1 states that the Newton polyhedron of $\Delta_{N_{G}}$ has a vertex $\lambda$ such that $c_{\lambda}= \pm 1$, dual to $\phi_{G}$. Define

$$
\lambda_{G}=\pi_{*} \lambda+ \begin{cases}0 & \text { if } b_{1}\left(N_{G}\right) \geq b_{1}(N)>1 \text { or } b_{1}\left(N_{G}\right)=b_{1}(N)=1, \\ \operatorname{div} \pi_{*} & \text { if } b_{1}\left(N_{G}\right)>b_{1}(N)=1 .\end{cases}
$$

We claim that $\lambda_{G}$ is a vertex of the Newton polyhedron of $\Delta_{N}^{\alpha}=\sum_{g \in H} b_{g} g$ such that $b_{\lambda_{G}}= \pm 1$. In fact by Theorem 2.1 $\phi\left(\pi_{*} \lambda\right)=\phi_{G}(\lambda)$ is the unique maximum over all elements in $\operatorname{supp} \Delta_{N_{G}} \subset H_{G}$, which entails that $\lambda_{G}$ is a vertex of $\operatorname{supp} \Delta_{N}^{\alpha}$, dual to $\phi$. Proposition 3.6 then implies that $b_{\lambda_{G}}=c_{\lambda}= \pm 1$.

As stated in Theorem 2.1 the vertex $\lambda$ equals $-\frac{1}{2} e_{F_{T, N_{G}}}$ if $b_{1}\left(N_{G}\right)>1$ and $-\frac{1}{2} e_{F_{T, N_{G}}}+1$ if $b_{1}\left(N_{G}\right)=1$. A straightforward calculation shows that $\pi_{*} e_{F_{T, N}}=|G| e_{F_{T, N}}$, which in turn implies the relation between $\lambda_{G}, e_{F_{T}}$ and $\lambda$ stated in (1). This, together with the equality $\varphi\left(-e_{F_{T}}\right)=\|\varphi\|_{T}$ that holds for all $\varphi \in \mathbb{R}_{+} F_{T}$, implies the equalities of (1).

The second part follows either from Theorem 2.1 (2) and Proposition 3.6 or it can be deduced from (1) as follows. Applying Theorem 3.5 as in Equation (5), we can write

$$
\Delta_{N, \phi}^{\alpha}= \begin{cases}\left(t^{\operatorname{div} \phi_{G}}-1\right)^{2} \phi\left(\Delta_{N}^{\alpha}\right)=\left(t^{\operatorname{div} \phi_{G}}-1\right)^{2}\left(\sum_{g \in H} b_{g} t^{\phi(g)}\right) & \text { if } b_{1}(N)>1, \\ \phi\left(\Delta_{N}^{\alpha}\right)=\sum_{g \in H} b_{g} t^{\phi(g)} & \text { if } b_{1}(N)=1 .\end{cases}
$$

In either case, by (1), the condition that $\phi$ is contained in $\mathbb{R}_{+} F_{T} \subset \mathbb{R}_{+} F_{A}^{\alpha}$ entails that $\phi(g)$ attains maximal value in correspondence of the unique $\lambda_{G} \in \operatorname{supp} \Delta_{N}^{\alpha}$, with coefficient $b_{\lambda_{G}}= \pm 1$. It follows that $\Delta_{N, \phi}^{\alpha}$ is monic and

$$
\operatorname{deg} \Delta_{N, \phi}^{\alpha}= \begin{cases}2 \phi\left(\lambda_{G}\right)+2 \operatorname{div} \phi_{G}=|G|\|\phi\|_{T}+2 \operatorname{div} \phi_{G} & \text { if } b_{1}(N)>1, \\ 2 \phi\left(\lambda_{G}\right)=|G|\|\phi\|_{T}+2 \operatorname{div} \phi_{G} & \text { if } b_{1}(N)=1,\end{cases}
$$

where for the last equality we have used part (1) and the relation $\operatorname{div} \phi_{G}=\operatorname{div} \pi_{*} \cdot \operatorname{div} \phi$. 
It is important to point out that the constraint of part (2) of Theorem 4.1 (and, later, its symplectic counterpart) is, in practice, the condition that can be directly tested to investigate if a class $\phi$ represents a fibration.

Our results in Section 5 and Section 7 (cf. also [FK06, Ch03, GKM05]) show that twisted Alexander polynomials are very efficient at detecting non-fibered manifolds. This leads us to conjecture the following:

Conjecture 4.2. Let $N$ be a 3-manifold and let $\phi \in H^{1}(N)$ a non-trivial class such that for any epimorphism onto a finite group $\alpha: \pi_{1}(N) \rightarrow G$ the twisted Alexander polynomial $\Delta_{N, \phi}^{\alpha} \in \mathbb{Z}\left[t^{ \pm 1}\right]$ is monic and $\operatorname{deg} \Delta_{N, \phi}^{\alpha}=|G|\|\phi\|_{T}+2 \operatorname{div} \phi_{G}$. Then $(N, \phi)$ fibers over $S^{1}$.

Note that this conjecture is slightly stronger than the converse of Theorem 4.1, as we are not requiring a priori that $\phi$ is in the cone over a top-dimensional face of the Thurston unit ball. In the next subsection, we will discuss how this conjecture relates to Conjecture 1.1. Further evidence to this conjecture is discussed in Section 8 .

Added in proof. In FV07. Conjecture 4.2 has been proved in the two special cases that $N$ has vanishing Thurston norm or that $N$ is a graph manifold.

4.2. Finite covers and symplectic manifolds. We can use the properties of Theorem 4.1 to further analyze the relation between fibered 3-manifolds and symplectic $S^{1} \times N$, to get more evidence to Conjecture 1.1. The proof of Theorem 4.1 boils down to the fact that finite covers of fibered manifolds are fibered again. Similarly, we will use the fact that finite covers of a symplectic manifold admit a symplectic structure to obtain our main theorem.

Theorem 4.3. Let $N$ be an irreducible 3 -manifold such that $S^{1} \times N$ admits a symplectic structure with Künneth component $\varphi \in H^{1}(N)$ and canonical class $K$. Then there exists a top-dimensional face $F_{T}$ of the Thurston unit ball such that $\varphi \in \mathbb{R}_{+} F_{T}$ for which the same properties as in (1) and (2) of Theorem 4.1 hold true, with $\lambda_{G}$ equal to $\frac{|G|}{2} P D_{N} K$ if $b_{1}(N)>1$ and $\frac{|G|}{2} P D_{N} K+\operatorname{div} \pi_{*}$ if $b_{1}(N)=1$.

Proof. First, observe that, denoting (with slight abuse of notation) the finite cover $S^{1} \times$ $N_{G} \rightarrow S^{1} \times N$ by $\pi$, the manifold $S^{1} \times N_{G}$ is symplectic with symplectic form $\pi^{*} \omega$ whose cohomology class has Künneth component $\pi^{*} \varphi=\varphi_{G}$, and canonical class $\pi^{*} K$ (that we can consider as an element of $H^{2}\left(N_{G}\right)$ ). Moreover, the assumption of irreducibility of $N$ implies that $N_{G}$ is irreducible too, by the Equivariant Sphere Theorem (cf. Du85]). Therefore we can apply Theorem 2.2 for $N_{G}$ in the same way we applied Theorem 2.1 in the proof of Theorem 4.1, with $\lambda$ equal to $\frac{1}{2} P D_{N_{G}} \pi^{*} K$ if $b_{1}\left(N_{G}\right)>1$ and equal to $\frac{1}{2} P D_{N_{G}} \pi^{*} K+1$ if $b_{1}\left(N_{G}\right)=1$. The class $\lambda_{G}$, defined as is Equation (7), is a vertex of the Newton polyhedron of $\Delta_{N}^{\alpha}$, dual to $\varphi$, with coefficient $b_{\lambda_{G}}= \pm 1$. The relation between the Thurston norm and the twisted Alexander norm of $\varphi$ follows verbatim that of Theorem 4.1, with $K$ playing the role of $-e_{F_{T, N}}$. To extend the result to the entire cone $\mathbb{R}_{+} F_{T}$, when $b_{1}(N) \geq 2$, we use the inclusion of the unit balls $B_{T} \subset|G| B_{A}^{\alpha}$ to grant that, as $F_{T}$ and $|G| F_{A}^{\alpha}$ intersect, $F_{T} \subset|G| F_{A}^{\alpha}$. Finally, to obtain (2), we proceed exactly as in the proof of Theorem 4.1,

For sake of clarity, we give a slightly weaker form of (2) of Theorem 4.3 that will provide, in what follows, the obstruction to the existence of symplectic forms which we mostly use. 
Proposition 4.4. Let $N$ be an irreducible 3-manifold such that $S^{1} \times N$ admits a symplectic structure with Künneth component $\phi \in H^{1}(N)$. Let $\alpha: \pi_{1}(N) \rightarrow G$ be an epimorphism onto a finite group, then $\Delta_{N, \phi}^{\alpha} \in \mathbb{Z}\left[t^{ \pm 1}\right]$ is monic and

$$
\operatorname{deg} \Delta_{N, \phi}^{\alpha}=|G|\|\phi\|_{T}+2 \operatorname{div} \phi_{G}
$$

Remark. (1) Note that (2) of Theorem 4.1 (respectively Proposition 4.4) implies that in order for a class $\phi$ to be fibered (respectively for $S^{1} \times N$ to admit a symplectic form with Künneth component $\phi$ ) the degrees of the twisted Alexander polynomials must satisfy $\operatorname{deg} \Delta_{N, \phi}^{\alpha}=|G| \operatorname{deg} \Delta_{N, \phi}+2\left(\operatorname{div} \pi_{*}-|G|\right) \operatorname{div} \phi$. This condition can be verified, for a given choice of $\alpha$, regardless of our explicit knowledge of the Thurston norm of $\phi$, on which we may have no information. In this sense, these results have a priori a larger field of application than Theorem 2.1.

(2) The content of Theorem 4.3 follows, ultimately, from the symplectic constraints on Seiberg-Witten invariants of finite covers of $S^{1} \times N$. In this sense, this result is just an application of the well-known principle that we can unveil information about a manifold by looking at the Seiberg-Witten invariants of its finite covers.

(3) It is not difficult to verify that, when $G=\mathbb{Z}_{d}$ and $\alpha: \pi_{1}(N) \rightarrow H \rightarrow \mathbb{Z}_{d}$ (i.e. when $N_{G}$ is a cyclic cover), Theorem 4.3 does not provide any new obstruction, as in that case the twisted Alexander polynomial is completely determined in terms of the ordinary Alexander polynomial.

(4) In [Ba01, the author asks whether the orbit space of any symplectic 4-manifold with a free $S^{1}$-action is fibered; an affirmative answer would include, in particular, Conjecture 1.1. It is possible that proceeding along the lines of Theorem $4.3 \mathrm{a}$ similar statement can be obtained.

In the next sections we will use these results to show the presence of new obstructions to the existence of symplectic structure on certain $S^{1} \times N$ 's for which the conditions of Theorem 2.2 are instead satisfied. But, perhaps more importantly, we can observe that analyzing the statement of Theorem 4.3 (or Proposition 4.4) we see that an irreducible $N$ for which $S^{1} \times N$ is symplectic satisfies the conditions of Conjecture 4.2. We have therefore the following:

Corollary 4.5. Conjecture 4.2 implies Conjecture 1.1 for irreducible 3-manifolds.

The condition of irreducibility can actually be removed assuming that the Geometrization Conjecture holds true, see Proposition 8.1. One appeal of Conjecture 4.2 is that it reduces Conjecture 1.1 to a purely 3-dimensional question (see also Section 8), that, although possibly stronger, has a distinctively more "quantitative" flavor than Conjecture 1.1. Note that, in fact, Conjecture 1.1 follows also from the slightly weaker condition that Conjecture 4.2 holds for the classes contained in a cone over the top-dimensional faces of the Thurston unit ball. In both cases Conjecture 1.1 is implied in the strongest sense, namely Conjecture 4.2 implies that the restriction of the symplectic cone(s) of $S^{1} \times N$ to $H^{1}(N ; \mathbb{R})$ coincides with the fibered cone(s) of $N$. 


\section{Abelian invariants do nOt Determine the EXistence of SympleCtiC STRUCTURES}

In this section we will exhibit a family of examples where, applying Theorem 4.3, we will be able to exclude the existence of symplectic structures, even though the ordinary Alexander polynomial does not provide any obstructions. These examples, in a sense, are defined $a d$ hoc to allow a simple computation of the twisted Alexander polynomial for suitable $G$. More challenging, and perhaps more interesting examples are discussed in Section 7 .

The examples we consider are obtained by 0-surgery on an infinite family of non-fibered knots $K_{i}$. Application of Theorem 4.3 is made possible by the fact that such manifolds, by Ga87, are irreducible. Actually, these examples allow us to show a stronger result, namely that abelian invariants of $K$ can not determine the existence of symplectic structures on a manifold of the form $S^{1} \times N(K)$. We have the following:

Theorem 5.1. Let $K$ be a non-trivial fibered knot. Then there exist infinitely many distinct knots $K_{i}, i \in \mathbb{N}$ such that for any $i$

(1) $\Delta_{K_{i}}=\Delta_{K}$, in particular $\Delta_{K_{i}}$ is monic,

(2) $\operatorname{deg}\left(\Delta_{K_{i}}\right)=2 g\left(K_{i}\right)$,

(3) $S^{1} \times N\left(K_{i}\right)$ is not symplectic,

(4) furthermore $N\left(K_{i}\right) \neq N\left(K_{j}\right)$ for any $i \neq j$.

The proof is somewhat similar to an argument of Cha in Ch03 concerning the existence of non-fibered knots with certain properties, and it will require the remainder of this section. Before proving this theorem, we briefly summarize the satellite construction for knots.

Let $K, C$ be knots. Let $A \subset S^{3} \backslash \nu K$ be a curve, unknotted in $S^{3}$. Then $S^{3} \backslash \nu A$ is a solid torus. Let $h: \partial(\overline{\nu A}) \rightarrow \partial(\overline{\nu C})$ be a diffeomorphism which sends a meridian of $A$ to a longitude of $C$, and a longitude of $A$ to a meridian of $C$. The space

$$
\left(S^{3} \backslash \nu A\right) \cup_{h}\left(S^{3} \backslash \nu C\right)
$$

is diffeomorphic to $S^{3}$. The image of $K$ is denoted by $S=S(K, C, A)$ and called a satellite knot. Note that we replaced a tubular neighborhood of $C$ by a knot in a solid torus, namely by $K \subset S^{3} \backslash \nu A$.

It is easy to see that $N(S)=(N(K) \backslash \nu A) \cup_{h}\left(S^{3} \backslash \nu C\right)$. By mapping $S^{3} \backslash \nu C$ to $\overline{\nu A}$ we get an induced map $N(S) \rightarrow N(K)$. Now let $\alpha: \pi_{1}(N(K)) \rightarrow G$ be a homomorphism to a finite group $G$; by the above we get an induced map $\pi_{1}(N(S)) \rightarrow G$. Note that $A$ defines an element in $[A] \in \pi_{1}(N(K))$ which is well-defined up to conjugation. Let $r$ be the order of $\alpha([A]) \in G$. Denote by $L(C)_{r}$ the $r$-fold cyclic cover of $S^{3}$ branched along $C$.

Lemma 5.2. Assume that $[A]=0 \in H_{1}\left(S^{3} \backslash \nu K\right)$. Then there exists a short exact sequence

$$
0 \rightarrow H_{1}\left(L(C)_{r}\right)^{\frac{|G|}{r}} \otimes_{\mathbb{Z}} \mathbb{Z}\left[t^{ \pm 1}\right] \rightarrow H_{1}\left(N(S) ; \mathbb{Z}[G]\left[t^{ \pm 1}\right]\right) \rightarrow H_{1}\left(N(K) ; \mathbb{Z}[G]\left[t^{ \pm 1}\right]\right) \rightarrow 0 .
$$

Proof. Consider the following commutative diagram of Mayer-Vietoris sequences of homology groups (where $R=\mathbb{Z}[G]\left[t^{ \pm 1}\right]$ ): 


$$
\begin{aligned}
& \rightarrow H_{1}(\partial(\overline{\nu A}) ; R) \rightarrow H_{1}(N(K) \backslash \nu A ; R) \oplus H_{1}\left(S^{3} \backslash \nu C ; R\right) \rightarrow H_{1}(N(S) ; R) \rightarrow 0
\end{aligned}
$$

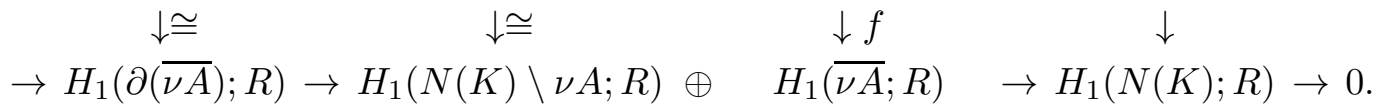

Note that $[A]=0 \in H_{1}\left(S^{3} \backslash \nu K\right)$ implies that $H_{1}\left(S^{3} \backslash \nu C\right) \rightarrow H_{1}(N(S))$ is the zero map; it follows that

$$
\begin{aligned}
H_{1}\left(S^{3} \backslash \nu C ; \mathbb{Z}[G]\left[t^{ \pm 1}\right]\right) & \cong H_{1}\left(\left(S^{3} \backslash \nu C\right)_{r}\right)^{\frac{|G|}{r}} \otimes_{\mathbb{Z}} \mathbb{Z}\left[t^{ \pm 1}\right], \\
H_{1}\left(\overline{\nu A} ; \mathbb{Z}[G]\left[t^{ \pm 1}\right]\right) & \cong \mathbb{Z}^{\frac{|G|}{r}} \otimes_{\mathbb{Z}} \mathbb{Z}\left[t^{ \pm 1}\right],
\end{aligned}
$$

where $\left(S^{3} \backslash \nu C\right)_{r}$ denotes the $r$-fold cyclic cover of $S^{3} \backslash \nu C$. Furthermore $H_{1}\left(\left(S^{3} \backslash \nu C\right)_{r}\right)=$ $H_{1}\left(L(C)_{r}\right) \oplus \mathbb{Z}$, where the $\mathbb{Z}$-summand is generated by the lift of $r$-copies of the meridian of $C$. It follows that

$$
\operatorname{Ker}\left\{H_{1}\left(S^{3} \backslash \nu C ; \mathbb{Z}[G]\left[t^{ \pm 1}\right]\right) \rightarrow H_{1}\left(\overline{\nu A} ; \mathbb{Z}[G]\left[t^{ \pm 1}\right]\right)\right\} \cong H_{1}\left(L(C)_{r}\right)^{\frac{|G|}{r}} \otimes_{\mathbb{Z}} \mathbb{Z}\left[t^{ \pm 1}\right] .
$$

The proof now follows easily from the above commutative diagram.

We are now in a position to prove Theorem 5.1 .

Proof of Theorem 5.1. Let $K$ be a non-trivial fibered knot with fiber $F$. Let $A \subset S^{3} \backslash$ $\nu K$ be a simple closed curve disjoint from $F$ that, after surgery, represents a non-trivial element in $\pi_{1}(N(K))^{(1)} / \pi_{1}(N(K))^{(2)}$. (Recall that the derived series of a group $\pi$ is defined inductively via $G^{(0)}=G, G^{(i+1)}=\left[G^{(i)}, G^{(i)}\right]$.) Note that we can find such an $A$ since $\pi_{1}\left(S^{3} \backslash\right.$ $K) \rightarrow \pi_{1}(N(K))$ is surjective and since $\pi_{1}(N(K))^{(1)} / \pi_{1}(N(K))^{(2)} \cong H_{1}\left(N(K) ; \mathbb{Z}\left[t^{ \pm 1}\right]\right) \cong$ $\mathbb{Z}^{2 \text { genus }(K)} \neq 0$. As the linking number of $A$ and $K$ is zero, $[A]$ is trivial in $H_{1}(N(K))$. After local crossing changes if necessary we can assume that $A$ is unknotted in $S^{3}$. As $N(K)$ is irreducible and since $b_{1}(N(K))=1$ it follows that $N(K)$ is Haken, hence $\pi_{1}(N(K))$ is residually finite (cf. [He87]). We can therefore find a finite group $G$ and an epimorphism $\alpha: \pi_{1}(N(K)) \rightarrow G$ such that $\alpha([A]) \neq e$. Denote by $r$ the order of $\alpha(A)$ in $G$. Note that $r>1$.

Let $C_{i}$ to be connected sum of $i$ copies of the figure -8 knot and of $i$ copies of the trefoil knot. It follows from Ch03, Lemma 4.4] that $H_{1}\left(L\left(C_{i}\right)_{r}\right)$ is non-trivial.

From Lemma 5.2 we have $H_{1}\left(N\left(K_{i}\right) ; \mathbb{Z}\left[t^{ \pm 1}\right]\right) \cong H_{1}\left(N(K) ; \mathbb{Z}\left[t^{ \pm 1}\right]\right)$, in particular $\Delta_{K_{i}}=$ $\Delta_{K}$. Also note that the image of $F$ in $\left(S^{3} \backslash \nu A\right) \cup_{\phi}\left(S^{3} \backslash \nu C_{i}\right)$ is still a Seifert surface for $K_{i}$ since $A \subset S^{3} \backslash F$, hence from Theorem 2.1 it follows that $2 g\left(K_{i}\right)=2 g(K)=\operatorname{deg}\left(\Delta_{K_{i}}\right)$.

We now show that $S^{1} \times N\left(K_{i}\right)$ is not symplectic. Let $\phi \in H^{1}\left(N\left(K_{i}\right)\right)$ be one of the two generators. By Lemma 5.2 there exists a short exact sequence

$$
0 \rightarrow H_{1}\left(L\left(C_{i}\right)_{r}\right)^{\frac{|G|}{r}} \otimes_{\mathbb{Z}} \mathbb{Z}\left[t^{ \pm 1}\right] \rightarrow H_{*}\left(N\left(K_{i}\right) ; \mathbb{Z}[G]\left[t^{ \pm 1}\right]\right) \rightarrow H_{1}\left(N(K) ; \mathbb{Z}[G]\left[t^{ \pm 1}\right]\right) \rightarrow 0 .
$$

Given a finitely generated $\mathbb{Z}\left[t^{ \pm 1}\right]$-module $H$ we can define its order ord $(H)$ as in Definition 3.1. By the multiplicativity of the order of $\mathbb{Z}\left[t^{ \pm 1}\right]$-modules (cf. [Le67, Lemma 5, p. 76]) we have

$$
\Delta_{N\left(K_{i}\right)}^{\alpha}=\Delta_{N(K)}^{\alpha} \text { ord }\left(H_{1}\left(L\left(C_{i}\right)_{r}\right)^{\frac{|G|}{r}} \otimes_{\mathbb{Z}} \mathbb{Z}\left[t^{ \pm 1}\right]\right)
$$


It is easy to see that

$$
\operatorname{ord}\left(H_{1}\left(L\left(C_{i}\right)_{r}\right)^{\frac{|G|}{r}} \otimes_{\mathbb{Z}} \mathbb{Z}\left[t^{ \pm 1}\right]\right)=\left|H_{1}\left(L\left(C_{i}\right)_{r}\right)\right|^{|G|} .
$$

It follows that $\Delta_{N\left(K_{i}\right)}^{\alpha}$ is not monic, hence $S^{1} \times N\left(K_{i}\right)$ is not symplectic by Theorem 4.3.

It remains to show that $N\left(K_{i}\right) \neq N\left(K_{j}\right)$ for $i \neq j$. Various methods are viable. We will distinguish the manifolds using the Cheeger-Gromov invariant [CG85] which associates to a pair $\left(N, \pi_{1}(N) \rightarrow H\right), H$ a group, a real number $\rho^{(2)}\left(N, \pi_{1}(N) \rightarrow H\right)$. Using standard arguments (cf. e.g. COT04]) one can show that

$$
\begin{aligned}
& \rho^{(2)}\left(N\left(K_{i}\right), \pi_{1}\left(N\left(K_{i}\right)\right) \rightarrow \pi_{1}\left(N\left(K_{i}\right)\right) / \pi_{1}\left(N\left(K_{i}\right)\right)^{(2)}\right) \\
= & \rho^{(2)}\left(N(K), \pi_{1}(N(K)) \rightarrow \pi_{1}(N(K)) / \pi_{1}(N(K))^{(2)}\right)+\rho^{(2)}\left(N\left(C_{i}\right), \pi_{1}\left(N\left(C_{i}\right)\right) \rightarrow \mathbb{Z}\right) .
\end{aligned}
$$

But $\rho^{(2)}\left(N\left(C_{i}\right), \pi_{1}\left(N\left(C_{i}\right)\right) \rightarrow \mathbb{Z}\right)$ equals the integral over the Levine-Tristram signature of $C_{i}$, which equals $\frac{4}{3} i$ (cf. e.g. COT04, Lemma 5.3]).

\section{THE MAIN THEOREM FOR FINITE FIELDS}

As we have seen in Section 5, under favorable circumstances Proposition 4.4 can be applied directly to verify that for certain manifolds $N, S^{1} \times N$ admits no symplectic structure. For other cases, however, there is no simple way to compute in a compact way twisted Alexander polynomials, and we are led to brute force calculations, based on Fox Calculus (see [CF77]). The goal of this section is to determine an effective method for implementing the results of Section 4 to get constraints that can be explicitly verified by a computer algebra system. As our main concern at this point is computational, we will focus only on the consequences of Theorem 4.3, but it should be clear that the results discussed in what follows can be formulated more or less verbatim for the study of fibered classes, partially recovering this way the results of [FK06].

The one-variable Alexander polynomial $\Delta_{\phi}^{\alpha} \in \mathbb{Z}\left[t^{ \pm 1}\right]$ can in theory be computed from a presentation of $\pi_{1}(N)$ using Fox calculus. In practice this turns out to be non viable since this requires the computation of determinants of large matrices over the ring $\mathbb{Z}\left[t^{ \pm 1}\right]$ and since it requires the computation of many greatest common divisors.

It is much easier to compute determinants and hence twisted Alexander polynomials over the principal ideal domain $\mathbb{F}_{p}\left[t^{ \pm 1}\right]$. As we will see switching to $\mathbb{F}_{p}\left[t^{ \pm 1}\right]$ also has the added advantage that we will be able to work with smaller representations (cf. Theorem 6.7).

Let $N$ be a 3 -manifold, $\alpha: \pi_{1}(N) \rightarrow G$ an epimorphism to a finite group, and let $\phi \in \operatorname{Hom}(H, F)$ be a non-trivial homomorphism. We denote the induced representation $\pi_{1}(N) \rightarrow \operatorname{Aut}_{\mathbb{Z}}(\mathbb{Z}[G])$ (cf. Section 3 for details) by $\alpha$ as well, and given a prime $p$ we denote the representation $\pi_{1}(N) \rightarrow \operatorname{Aut}_{\mathbb{F}_{p}}\left(\mathbb{F}_{p}[G]\right)$ by $\alpha_{p}$. We therefore get Alexander polynomials $\Delta^{\alpha} \in \mathbb{Z}[F]$ and $\Delta^{\alpha_{p}} \in \mathbb{F}_{p}[F]$. Remember that $\Delta^{\alpha_{p}} \in \mathbb{F}_{p}[F]$ is well-defined only up to an overall multiplication by a non-zero element of $\mathbb{F}_{p}$, see Section 3 . In particular we lose the notion of monicness. This polynomial is related to $\Delta^{\alpha}$ by the following proposition, which is another direct corollary to Lemma 3.4 and Theorem 3.5 .

Proposition 6.1. Let $\phi: H \rightarrow F$ be a non-trivial homomorphism to a free abelian group $F$. Let $\Delta^{\alpha} \in \mathbb{Z}[F]$ and $\Delta^{\alpha_{p}} \in \mathbb{F}_{p}[F]$ be the twisted Alexander polynomials associated to an 
epimorphism $\alpha: \pi_{1}(N) \rightarrow G$. Then

$$
\Delta^{\alpha_{p}}=\Delta^{\alpha} \bmod p
$$

where the relation above holds in $\mathbb{F}_{p}[F]$ up to multiplication by a unit in $\mathbb{F}_{p}[F]$.

Proposition 6.1 provides a way to determine, at least in principle, the twisted Alexander norm $\|\cdot\|_{A}^{\alpha}$ in terms of the family of norms $\|\cdot\|_{A}^{\alpha_{p}}$ associated to the twisted Alexander polynomials $\Delta^{\alpha_{p}}$ since Equation (10) implies that $\operatorname{supp} \Delta^{\alpha_{p}} \subset \operatorname{supp} \Delta^{\alpha}$ and $\bigcup_{p} \operatorname{supp} \Delta^{\alpha_{p}}=$ $\operatorname{supp} \Delta^{\alpha}$. Furthermore Proposition 6.1 allows us to characterize the elements of $\operatorname{supp} \Delta^{\alpha}$ with coefficient \pm 1 , as these are the only ones whose reduction $\bmod p$ is never trivial. In sum, we have the following corollary:

Corollary 6.2. Let $N$ be a closed 3-manifold and let $\alpha: \pi_{1}(N) \rightarrow G$ an epimorphism to a finite group. Then for all $\phi \in H^{1}(N ; \mathbb{R})$

$$
\|\phi\|_{A}^{\alpha}=\max _{p}\left\{\|\phi\|_{A}^{\alpha_{p}}\right\} .
$$

Furthermore if $\phi$ lies in the cone over a top-dimensional face of the unit ball of the twisted Alexander norm $\|-\|_{A}^{\alpha}$ which is dual to an element of supp $\Delta^{\alpha}$ with coefficient \pm 1 , then $\|\phi\|_{A}^{\alpha}=\|\phi\|_{A}^{\alpha_{p}}$ for all primes $p$.

Using Corollary 6.2, we can write a reformulation of Theorem 4.3 for finite fields:

Theorem 6.3. Let $N$ be an irreducible 3 -manifold such that $S^{1} \times N$ admits a symplectic structure with Künneth component $\varphi \in H^{1}(N)$. Then there exists a top-dimensional face $F_{T}$ of the Thurston unit ball such that $\varphi \in \mathbb{R}_{+} F_{T}$. Furthermore for any prime $p$ we have $\Delta^{\alpha_{p}} \neq 0$ and for all $\phi \in \mathbb{R}_{+} F_{T} \cap H^{1}(N)$ we have

$$
\|\phi\|_{A}^{\alpha_{p}}=|G|\|\phi\|_{T}+ \begin{cases}0 & \text { if } b_{1}(N)>1, \\ 2 \operatorname{div} \phi_{G} & \text { if } b_{1}(N)=1 .\end{cases}
$$

In particular, if $\phi \in \mathbb{R}_{+} F_{T} \cap H^{1}(N)$, deg $\Delta_{\phi}^{\alpha_{p}}=|G|\|\phi\|_{T}+2$ div $\phi_{G}$ for all primes $p$.

Remark. (1) Note that, due to the indeterminacy in the definition of the coefficients of $\Delta^{\alpha_{p}}$, condition (1) in Theorem 4.3 implies just that $\lambda_{G}$ has non-zero coefficient in $\Delta^{\alpha_{p}}$. In this sense, that condition is automatically included in Equation (11). On the other direction, the non-vanishing of the coefficient of $\lambda_{G}$ in $\Delta^{\alpha_{p}}$ for all primes $p$ (implied by Equation (11)) guarantees that the coefficient of $\lambda_{G}$ in $\Delta^{\alpha}$ equals \pm 1 .

(2) Theorem 6.3 ensures that if $N$ is an irreducible manifold and $\phi \in \mathbb{R}_{+} F_{T} \cap H^{1}(N)$ represents the Künneth component of a symplectic form, then $\Delta_{\phi}^{\alpha_{p}}$ is non-trivial for all primes $p$.

At this point, we could implement Theorem 6.3 by computing the polynomials $\Delta_{\phi}^{\alpha_{p}}$ using Fox calculus. However, the computations would still be very time consuming since the matrices involved are very large. It is therefore much more useful to find criteria involving 'small' representations of $\pi_{1}(N)$ : Assume we are given a representation $\beta_{p}: G \rightarrow \operatorname{Aut}_{\mathbb{F}_{p}}(B)$, $B$ a finite $\mathbb{F}_{p}$-module. We have Alexander polynomials $\Delta_{N, \phi, i}^{\beta_{p} \circ \alpha} \in \mathbb{F}_{p}\left[t^{ \pm 1}\right]$ which we denote by $\Delta_{N, \phi, i}^{\beta_{p}}$. It is well-known that given a presentation of $\pi_{1}(N)$ these polynomials can be 
computed for $i=0,1$ using Fox calculus; we refer to [FK06 for the computation when $i=2$.

For these Alexander polynomials, a McMullen-type inequality (similar to Lemma 3.9) is one of the main results of [FK05] and [FK06]:

Theorem 6.4. Let $N$ be a 3-manifold, $B$ a finite $\mathbb{F}_{p}$-module and let $\beta_{p}: G \rightarrow$ Aut $\mathbb{F}_{p}(B)$ be a representation. If $\Delta^{\beta_{p}} \neq 0$, then for all non-zero $\phi \in H^{1}(N)$ we have the following inequalities:

$$
\|\phi\|_{A}^{\beta_{p}} \leq \operatorname{dim}(B)\|\phi\|_{T}+ \begin{cases}0 & \text { if } b_{1}(N)>1, \\ \operatorname{deg} \Delta_{\phi, 0}^{\beta_{p}}+\operatorname{deg} \Delta_{\phi, 2}^{\beta_{p}} & \text { if } b_{1}(N)=1 .\end{cases}
$$

Note that in the case where $B=\mathbb{F}_{p}[G]$ we can apply Lemma 3.4 to conclude that $\operatorname{div} \phi_{G}=\operatorname{deg} \Delta_{\phi, 0}^{\alpha_{p}}=\operatorname{deg} \Delta_{\phi, 2}^{\alpha_{p}}$.

The constraints on the existence of symplectic structures obtained thus far (Theorems 4.3 and 6.3) apply to the twisted Alexander polynomials defined using the modules $\mathbb{Z}[G][H]$ and $\mathbb{F}_{p}[G][H]$. We want to translate them into conditions on the polynomials $\Delta_{N, \phi, i}^{\beta_{p}}$. To do so, we will need a further assumption, namely we will choose $\mathbb{F}_{p}$ and $G$ so that any $\mathbb{F}_{p}[G]$-module is semisimple, i.e. that the $\mathbb{F}_{p}$-representations of $G$ are completely reducible. Concerning this we have the following well-known version of Maschke's Theorem:

Theorem 6.5. Let $p$ be a prime such that $(p,|G|)=1$. Then every $\mathbb{F}_{p}[G]$-module is semisimple. Moreover, every simple $\mathbb{F}_{p}[G]$-module is a direct summand of $\mathbb{F}_{p}[G]$.

Proof. For the first part we refer to [Ro96, p. 216]. For the second part, let $C$ be a simple $\mathbb{F}_{p}[G]$-module and let $v \in C$. Then there exists a short exact sequence of $\mathbb{F}_{p}[G]$-modules:

$$
\begin{aligned}
0 \rightarrow K \rightarrow \mathbb{F}_{p}[G] & \rightarrow C \rightarrow 0 \\
g & \mapsto v g .
\end{aligned}
$$

Note that the last map must be an epimorphism since $C$ is simple. Since $\mathbb{F}_{p}[G]$ is a semisimple left $\mathbb{F}_{p}[G]$-module, the sequence above splits and we can write $\mathbb{F}_{p}[G]=K \oplus C$.

Let us then assume that $(p,|G|)=1$. The twisted Alexander polynomials associated to a reducible $\mathbb{F}_{p}[G]$-module satisfy the following lemma.

Lemma 6.6. Let $\beta_{p}: G \rightarrow A u t_{\mathbb{F}_{p}}(B)$ and $\gamma_{p}: G \rightarrow A u t_{\mathbb{F}_{p}}(C)$ be finite dimensional representations. Denote the direct sum of the representations by $\beta_{p} \oplus \gamma_{p}: G \rightarrow A u t_{\mathbb{F}_{p}}(B \oplus C)$. Furthermore let $\phi: \pi_{1}(N) \rightarrow F$ be a non-trivial homomorphism to a free abelian group $F$. Then

$$
\Delta_{\phi, i}^{\beta_{p} \oplus \gamma_{p}}=\Delta_{\phi, i}^{\beta_{p}} \cdot \Delta_{\phi, i}^{\gamma_{p}}
$$

and, for all $\phi \in H^{1}(N ; \mathbb{R}),\|\phi\|_{A}^{\beta_{p} \oplus \gamma_{p}}=\|\phi\|_{A}^{\beta_{p}}+\|\phi\|_{A}^{\gamma_{p}}$.

Proof. We have $H_{*}(N ;(B \oplus C)[F]) \cong H_{*}(N ; B[F]) \oplus H_{*}(N ; C[F])$; therefore we can get a free resolution of $H_{*}(N ;(B \oplus C)[F])$ as direct sum of the resolutions of $H_{*}(N ; B[F])$ and $H_{*}(N ; C[F])$, which implies the relation between the twisted Alexander polynomials. The relation between the twisted Alexander norms follows at once from that relation (for the case of $F=H)$, for example by looking at the dense subset of $H^{1}(N ; \mathbb{R})$ given by the 
intersection of the cones on the top-dimensional faces of the unit balls of the norms $\|\cdot\|_{A}^{\beta_{p}}$ and $\|\cdot\|_{A}^{\gamma_{p}}$, and using continuity.

We can state now the analogue of Theorem 6.3 for a general $\mathbb{F}_{p}[G]$-module $B$ with $(p,|G|)=$ 1:

Theorem 6.7. Let $N$ be an irreducible 3 -manifold such that $S^{1} \times N$ admits a symplectic structure with Künneth component $\varphi \in H^{1}(N)$; then there exists a top-dimensional face $F_{T}$ of the Thurston unit ball such that $\varphi \in \mathbb{R}_{+} F_{T}$ and such that for all $\phi \in \mathbb{R}_{+} F_{T} \cap H^{1}(N)$, any epimorphism $\alpha: \pi_{1}(N) \rightarrow G$ onto a finite group $G$ and any $\mathbb{F}_{p}[G]$-module $B$ with $(p,|G|)=1$

$$
\|\phi\|_{A}^{\beta_{p}}=\operatorname{dim}(B)\|\phi\|_{T}+ \begin{cases}0 & \text { if } b_{1}(N)>1, \\ \operatorname{deg} \Delta_{\phi, 0}^{\beta_{p}}+\operatorname{deg} \Delta_{\phi, 2}^{\beta_{p}} & \text { if } b_{1}(N)=1 .\end{cases}
$$

In particular, if $\phi \in \mathbb{R}_{+} F_{T} \cap H^{1}(N)$, $\operatorname{deg} \Delta_{\phi}^{\beta_{p}}=\operatorname{dim}(B)\|\phi\|_{T}+\operatorname{deg} \Delta_{\phi, 0}^{\beta_{p}}+\operatorname{deg} \Delta_{\phi, 2}^{\beta_{p}}$ for all primes $p$.

Proof. First observe that by Maschke's Theorem and Lemma 6.6, we can limit ourselves to consider the case where $B$ is a simple $\mathbb{F}_{p}[G]$-module, and thus a direct summand of $\mathbb{F}_{p}[G]$. Denote by $C$ the $\mathbb{F}_{p}[G]$-module such that $\mathbb{F}_{p}[G]=B \oplus C$. Denote the $\mathbb{F}_{p}[G]$-module structures by $\beta_{p}$ respectively $\gamma_{p}$. Note that it follows from Theorem 6.3 and Lemma 6.6 that $\Delta^{\beta_{p}} \neq 0$ and $\Delta^{\gamma_{p}} \neq 0$, which allows us to apply Theorem 6.4. Now applying in order Theorem 6.3, Lemma 6.6 and Theorem 6.4 (twice) we have

$$
\begin{aligned}
& |G|\|\phi\|_{T}+\left\{\begin{array}{l}
0 \\
\operatorname{deg} \Delta_{\phi, 0}^{\alpha_{p}}+\operatorname{deg} \Delta_{\phi, 2}^{\alpha_{p}}
\end{array}=\|\phi\|_{A}^{\alpha_{p}}=\|\phi\|_{A}^{\beta_{p}}+\|\phi\|_{A}^{\gamma_{p}} \leq\right. \\
& \leq\|\phi\|_{A}^{\beta_{p}}+\operatorname{dim}(C)\|\phi\|_{T}+ \begin{cases}0 & \text { if } b_{1}(N)>1 \\
\operatorname{deg} \Delta_{\phi, 0}^{\gamma_{p}}+\operatorname{deg} \Delta_{\phi, 2}^{\gamma_{p}} & \text { if } b_{1}(N)=1\end{cases} \\
& \leq(\operatorname{dim}(B)+\operatorname{dim}(C))\|\phi\|_{T}+ \begin{cases}0 & \text { if } b_{1}(N)>1, \\
\operatorname{deg} \Delta_{\phi, 0}^{\beta_{p}}+\operatorname{deg} \Delta_{\phi, 2}^{\beta_{p}}+\operatorname{deg} \Delta_{\phi, 0}^{\gamma_{p}}+\operatorname{deg} \Delta_{\phi, 2}^{\gamma_{p}} & \text { if } b_{1}(N)=1 .\end{cases}
\end{aligned}
$$

It follows from Lemma 6.6 that the last term equals the first term. But this implies that all inequalities are in fact equalities, and this shows that (12) holds for a simple module, and thus for all $\mathbb{F}_{p}[G]$-modules. Finally, the condition on $\operatorname{deg} \Delta_{\phi}^{\beta_{p}}$ follows from (12), a version of Theorem 3.5 for general representations proved in [FK05] and the argument in the proof of Proposition 3.8, applied to $\Delta^{\beta_{p}}$.

Remark. In studying the problem of fiberability of $(N, \phi)$ we could have obtained the result of Theorem 6.7 directly for any $R[G]$-module, without having to pass through the results of Section 4 (this is in fact the approach of [FK06]). Instead, for the symplectic case, we have to follow that path, as the twisted Alexander polynomial $\Delta^{\alpha}$ associated to $R[G]$ is 
the only one that can be interpreted directly in terms of Seiberg-Witten theory, allowing us to apply the corresponding symplectic constraints. It is entirely possible, however, that Theorem 6.7 holds true without the condition $(p,|G|)=1$.

\section{KRONHEIMER'S EXAMPLE AND 12-CROSSING KNOTS}

7.1. Kronheimer's example: the Pretzel knot $P=(5,-3,5)$. We will now consider the Pretzel knot $P=(5,-3,5)$, whose diagram is given in Figure1, This knot has Alexander polynomial $t^{2}-3 t+1$ and has a Seifert surface of genus one. The fundamental group of

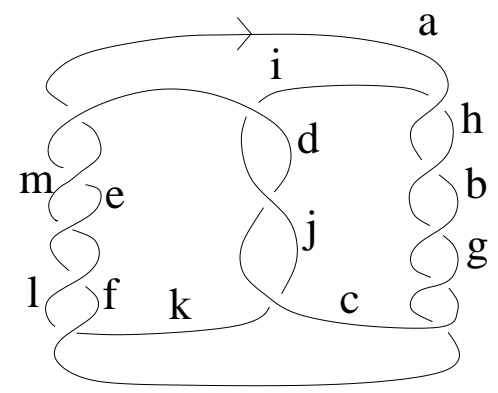

Figure 1. The pretzel knot $(5,-3,5)$.

$S^{3} \backslash \nu P$ is generated by the meridians $a, b, \ldots, m$ of the strands in the knot diagram of Figure 1, The relations are

$$
\begin{aligned}
& a=h^{-1} b h, \quad b=g^{-1} c g, \quad c=j d j^{-1}, \quad d=m^{-1} e m, \quad e=l^{-1} f l, \\
& f=c^{-1} g c, \quad g=b^{-1} h b, \quad h=a^{-1} i a, \quad i=d^{-1}, \quad j=c k c^{-1}, \\
& k=f^{-1} l f, \quad l=e^{-1} m e, \quad m=d^{-1} a d .
\end{aligned}
$$

The longitude is represented by $\lambda=h^{-1} g^{-1} j m^{-1} l^{-1} c^{-1} b^{-1} a^{-1} d c f^{-1} e^{-1} d^{-1} a^{7}$. Clearly $\pi_{1}(N(P))=\pi_{1}\left(S^{3} \backslash \nu P\right) /\langle\lambda\rangle$.

Using the computer program KnotTwister [Fr05] we found an epimorphism $\alpha: \pi_{1}(N(P)) \rightarrow$ $S_{5}$ which is given by

$$
\begin{aligned}
& a \mapsto \text { (51234) } \quad b \mapsto \text { (43521) } \quad c \quad \mapsto \text { (54132) } \quad d \mapsto \text { (43521) } \\
& e \mapsto(35214) \quad f \mapsto \text { (23451) } g \mapsto(35214) \quad h \mapsto \text { (24153) } \\
& i \mapsto(35421) \quad j \mapsto \text { (41532) } \quad k \mapsto \text { (54213) } l \mapsto \text { (41532) } \\
& m \mapsto \text { (24153). }
\end{aligned}
$$

Now consider the representation $\beta_{7}: \pi_{1}(N(P)) \stackrel{\alpha}{\rightarrow} S_{5} \rightarrow \mathrm{GL}\left(\mathbb{F}_{7}, 5\right)$ where $S_{5}$ acts on $\mathbb{F}_{7}^{5}$ by permutation of the coordinates. Using KnotTwister we compute that $\Delta^{\beta_{7}}=0$ and $\operatorname{deg} \Delta_{0}^{\beta_{7}}=\operatorname{deg} \Delta_{2}^{\beta_{7}}=1$. Since $p=7$ and $\left|S_{5}\right|=120$ are coprime it follows immediately from Theorem 6.7, applied to a generator $\phi \in H^{1}(N(P))$, that $S^{1} \times N(P)$ is not symplectic.

7.2. 12-crossing knots. It is well-known that a knot $K$ with 11 crossings or less is fibered (and equivalently $N(K)$ is fibered) if and only if $2 g(K)=\operatorname{deg}\left(\Delta_{K}\right)$ and $\Delta_{K}$ is monic. It follows therefore from Theorem 2.2 that if $K$ is a knot with 11 crossings or less, then $S^{1} \times N(K)$ is symplectic if and only if $K$ is fibered. 
By work of Stoimenow and Hirasawa and the results of [FK06] there exist 13 non-fibered 12 - crossing knots which have monic Alexander polynomials and such that $\operatorname{deg}\left(\Delta_{K}\right)=$ $2 g(K)$. According to [FK06] these knots are 12 $1345,12_{1498}, 12_{1502}, 12_{1546}, 12_{1567}, 12_{1670}$, $12_{1682}, 12_{1752}, 12_{1771}, 12_{1823}, 12_{1938}, 12_{2089}, 12_{2103}$ (where we use Knotscape notation [HT]). For these 13 knots Theorem 2.2 is not strong enough to show that $S^{1} \times N(K)$ is not symplectic. The following table shows all the knots for which we found a representation $\beta_{p}: \pi_{1}(N(K)) \rightarrow G=S_{k} \rightarrow \mathrm{GL}\left(\mathbb{F}_{p}, k\right)$ (where $S_{k}$ acts by permutation on $\mathbb{F}_{p}^{k}$ ) such that $\operatorname{gcd}\left(p,\left|S_{k}\right|\right)=1$ and such that either $\Delta^{\beta_{p}}=0$ or

$$
\operatorname{deg} \Delta^{\beta_{p}}<k \cdot(2 g(K)-2)+\operatorname{deg} \Delta_{0}^{\beta_{p}}+\operatorname{deg} \Delta_{2}^{\beta_{p}} .
$$

For these knots $K$ the manifold $S^{1} \times N(K)$ is not symplectic by Theorem6.7. In the following table we give all the relevant data. Recall that we write $\operatorname{deg} \Delta^{\beta_{p}}=-\infty$ if $\Delta^{\beta_{p}}=0$.

\begin{tabular}{|c|c|c|c|c|c|c|}
\hline Knot & $12_{1345}$ & $12_{1498}$ & $12_{1502}$ & $12_{1546}$ & $12_{1567}$ & $12_{1752}$ \\
\hline$\|\phi\|_{T}=2 g(K)-2$ & 2 & 4 & 4 & 2 & 2 & 2 \\
\hline Order $k$ of permutation group & 6 & 6 & 5 & 5 & 5 & 6 \\
\hline Prime $p$ & 7 & 7 & 11 & 7 & 7 & 17 \\
\hline $\operatorname{deg} \Delta_{0}^{\beta_{p}}=\operatorname{deg} \Delta_{2}^{\beta_{p}}$ & 1 & 1 & 1 & 1 & 1 & 1 \\
\hline $\operatorname{deg} \Delta^{\beta_{p}}$ & $-\infty$ & 24 & 14 & $-\infty$ & $-\infty$ & 10 \\
\hline$k(2 g(K)-2)+\operatorname{deg} \Delta_{0}^{\beta_{p}}+\operatorname{deg} \Delta_{2}^{\beta_{p}}$ & 14 & 26 & 22 & 12 & 12 & 14 \\
\hline Knot & $12_{1670}$ & $12_{1771}$ & $12_{1823}$ & $12_{1938}$ & $12_{2089}$ & $12_{2103}$ \\
\hline$\|\phi\|_{T}=2 g(K)-2$ & 2 & 2 & 2 & 2 & 2 & 2 \\
\hline Order $k$ of permutation group & 6 & 5 & 6 & 5 & 5 & 5 \\
\hline Prime $p$ & 17 & 7 & 7 & 11 & 11 & 7 \\
\hline $\operatorname{deg} \Delta_{0}^{\beta_{p}}=\operatorname{deg} \Delta_{2}^{\beta_{p}}$ & 1 & 2 & 2 & 1 & 1 & 1 \\
\hline $\operatorname{deg} \Delta^{\tilde{\beta}_{p}}$ & 10 & 10 & $-\infty$ & 4 & 4 & $-\infty$ \\
\hline$k(2 g(K)-2)+\operatorname{deg} \Delta_{0}^{\beta_{p}}+\operatorname{deg} \Delta_{2}^{\beta_{p}}$ & 14 & 16 & 16 & 14 & 14 & 14 \\
\hline
\end{tabular}

This approach did not work for the knot $K=12_{1682}$. The stumbling block in our efforts was the condition $(|G|, p)=1$. However, we can bypass this problem by applying directly Theorem 6.3 using a map $\alpha: \pi_{1}(N(K)) \rightarrow A_{4}$ such that $\Delta^{\alpha_{3}} \in \mathbb{F}_{3}\left[t^{ \pm 1}\right]$ has degree 21 and $\operatorname{deg}\left(\Delta_{0}^{\alpha_{3}}\right)=\operatorname{deg}\left(\Delta_{2}^{\alpha_{3}}\right)=3$. Note that $\left|A_{4}\right|=12$ and that $g(K)=2$. It follows that

$$
\operatorname{deg} \Delta^{\alpha_{3}}=21 \neq 30=\left|A_{4}\right|(2 g(K)-2)+\operatorname{deg} \Delta_{0}^{\alpha_{3}}+\operatorname{deg} \Delta_{2}^{\alpha_{3}} .
$$

Therefore $S^{1} \times N(K)$ is not symplectic by Theorem 6.3. Together with the previous results, we conclude that Conjecture 1.1 holds for all knots with up to 12 crossings.

Remark. Note that the proof of non-fiberedness of these knots contained in [FK06] does not, in many cases, apply to show that $S^{1} \times N(K)$ is not symplectic, as the (computationally somewhat simpler) representations therein considered mostly fail to satisfy the condition $(p,|G|)=1$.

\section{The FIBEREDNESS CONJECTURE AND 3-MANiFOLD GROUPS}

The aim of this section is to discuss some evidence to Conjecture 4.2. To start, it is more or less explicitly known that the hypothesis of Conjecture 4.2 are sufficient (assuming the 
Geometrization Conjecture) to show that $N$ is prime, a condition that is well-known to hold for fibered manifolds. Precisely, we have the following:

Proposition 8.1. Let $N$ be a 3-manifold which is not prime and let $\phi \in H^{1}(N)$. If the Geometrization Conjecture holds, then there exists an epimorphism $\alpha: \pi_{1}(N) \rightarrow G, G$ a finite group, such that $\Delta_{\phi}^{\alpha}(t)=0 \in \mathbb{Z}\left[t^{ \pm 1}\right]$.

This statement affords a direct proof using a Mayer-Vietoris decomposition for the Alexander module for a connected sum. It can also be simply deduced (see McC01 or [Vi99]) from standard vanishing theorems for Seiberg-Witten invariants of a suitable cover of $N$, whose existence is granted by the Geometrization Conjecture, which implies that $\pi_{1}(N)$ is residually finite (cf. [He87]). Note that without that assumption, the proof shows that a manifold $N$ satisfying the hypothesis of Conjecture 4.2 is prime up to the connected sum with a homology sphere which does not admit non-trivial finite covers.

In the following let $N$ be a $3-$ manifold and let $\phi \in H^{1}(N)$ a primitive class such that for any epimorphism onto a finite group $\alpha: \pi_{1}(N) \rightarrow G$ the twisted Alexander polynomial $\Delta_{N, \phi}^{\alpha} \in \mathbb{Z}\left[t^{ \pm 1}\right]$ is monic and $\operatorname{deg} \Delta_{N, \phi}^{\alpha}=|G|\|\phi\|_{T}+2 \operatorname{div} \phi_{G}$. Let $S$ be a Thurston norm minimizing surface dual to $\phi$. As by hypothesis $\Delta_{\phi} \neq 0$ we can assume, by [McM02], that $S$ is connected. Then let $M$ be the result of cutting $N$ along $S$. Denote the positive and the negative inclusions of $S$ into $M$ by $i_{+}$and $i_{-}$. Since $S$ is minimal, $i_{+}: \pi_{1}(S) \rightarrow \pi_{1}(M)$ is injective by Dehn's Lemma. Since $N$ is prime it follows easily from Stallings' theorem St62 that $(N, \phi)$ fibers over $S^{1}$ if and only if $i_{+}: \pi_{1}(S) \rightarrow \pi_{1}(M)$ is surjective.

Proposition 8.2. $H_{1}^{\alpha}(S ; \mathbb{Z}[G])$ and $H_{1}^{\alpha}(M ; \mathbb{Z}[G])$ are free abelian groups of the same rank.

Proof. First note that $H_{1}^{\alpha}(S ; \mathbb{Z}[G])$ is the first homology of the (not necessarily connected) cover of $S$ corresponding to $\pi_{1}(S) \rightarrow G$, hence $H_{1}^{\alpha}(S ; \mathbb{Z}[G])$ is free abelian.

Let $p$ be a prime. It follows from Proposition 6.1 that $\Delta_{\phi}^{\alpha_{p}} \neq 0$. By Lemma 3.4 $H_{2}^{\alpha}\left(N ; \mathbb{F}_{p}[G]\left[t^{ \pm 1}\right]\right)$ is $\mathbb{F}_{p}\left[t^{ \pm 1}\right]$-torsion. Therefore the Mayer-Vietoris sequence for $N$ with $\mathbb{F}_{p}[G]\left[t^{ \pm 1}\right]$-coefficients gives a short exact sequence (cf. [FK06] for details)

$0 \rightarrow H_{1}^{\alpha_{p}}\left(S ; \mathbb{F}_{p}[G]\right) \otimes_{\mathbb{F}_{p}} \mathbb{F}_{p}\left[t^{ \pm 1}\right] \stackrel{t i_{+}-i_{-}}{\longrightarrow} H_{1}^{\alpha_{p}}\left(M ; \mathbb{F}_{p}[G]\right) \otimes_{\mathbb{F}_{p}} \mathbb{F}_{p}\left[t^{ \pm 1}\right] \rightarrow H_{1}^{\alpha_{p}}\left(N ; \mathbb{F}_{p}[G]\left[t^{ \pm 1}\right]\right) \rightarrow 0$

Tensoring with $\mathbb{F}_{p}(t)$ we see that in particular $H_{1}^{\alpha_{p}}\left(S ; \mathbb{F}_{p}[G]\right) \cong H_{1}^{\alpha_{p}}\left(M ; \mathbb{F}_{p}[G]\right)$ for every prime $p$ as $\mathbb{F}_{p}$-vector spaces. Note that $H_{0}^{\alpha}(S ; \mathbb{Z}[G]) \cong \mathbb{Z}\left[G / \operatorname{Im}\left\{\pi_{1}(S) \rightarrow G\right\}\right]$ and $H_{0}^{\alpha}(M ; \mathbb{Z}[G]) \cong \mathbb{Z}\left[G / \operatorname{Im}\left\{\pi_{1}(M) \rightarrow G\right\}\right] ;$ in particular both are $\mathbb{Z}$-torsion free. It follows from the universal coefficient theorem applied to the $\mathbb{Z}$-module complex $C_{*}(\tilde{S}) \otimes_{\mathbb{Z}\left[\pi_{1}(S)\right]} \mathbb{Z}[G]$ that

$$
H_{1}^{\alpha}(S ; \mathbb{Z}[G]) \otimes_{\mathbb{Z}} \mathbb{F}_{p} \cong H_{1}^{\alpha_{p}}\left(S ; \mathbb{F}_{p}[G]\right)
$$

for every prime $p$. The same statement holds for $M$. Combining our results we see that $H_{1}^{\alpha}(S ; \mathbb{Z}[G]) \otimes_{\mathbb{Z}} \mathbb{F}_{p} \cong H_{1}^{\alpha}(M ; \mathbb{Z}[G]) \otimes_{\mathbb{Z}} \mathbb{F}_{p}$ for any prime $p$. It follows that $H_{1}^{\alpha}(S ; \mathbb{Z}[G]) \cong$ $H_{1}^{\alpha}(M ; \mathbb{Z}[G])$.

Now consider the exact sequence

$$
H_{1}^{\alpha}(S ; \mathbb{Z}[G]) \otimes \mathbb{Z}\left[t^{ \pm 1}\right] \stackrel{t i_{+}-i_{-}}{\longrightarrow} H_{1}^{\alpha}(M ; \mathbb{Z}[G]) \otimes \mathbb{Z}\left[t^{ \pm 1}\right] \rightarrow H_{1}^{\alpha}\left(N ; \mathbb{Z}[G]\left[t^{ \pm 1}\right]\right) \rightarrow 0 .
$$


Since $H_{1}^{\alpha}(S ; \mathbb{Z}[G])$ and $H_{1}^{\alpha}(M ; \mathbb{Z}[G])$ are free abelian groups of the same rank it follows that $\Delta_{\phi}^{\alpha}(t)=\operatorname{det}\left(t i_{+}-i_{-}\right)$. Note that this does not yet imply that $i_{+}$and $i_{-}$have full rank. Write $b_{i}^{\alpha}(S)=\operatorname{rank}_{\mathbb{Z}}\left(H_{i}^{\alpha}(S ; \mathbb{Z}[G])\right)$. A standard Euler characteristic argument shows that

$$
-b_{0}^{\alpha}(S)+b_{1}^{\alpha}(S)-b_{2}^{\alpha}(S)=-|G| \chi(S)=|G| \cdot\|\phi\|_{T} .
$$

Furthermore $b_{i}^{\alpha}(S)=\operatorname{deg} \Delta_{\phi, i}^{\alpha}$ for $i=1,2$ by [FK06, Propositions 4.9 and 4.11]. Combining this with the assumption

$$
\operatorname{deg} \Delta_{\phi}^{\alpha}=|G|\|\phi\|_{T}+2 \operatorname{div} \phi_{G}=|G|\|\phi\|_{T}+\operatorname{deg} \Delta_{\phi, 0}^{\alpha}+\operatorname{deg} \Delta_{\phi, 2}^{\alpha}
$$

we get that $\operatorname{deg}\left(\operatorname{det}\left(t i_{+}-i_{-}\right)\right)=\operatorname{deg} \Delta_{\phi}^{\alpha}=b_{1}^{\alpha}(S)$. Since $i_{+}$and $i_{-}$are $b_{1}^{\alpha}(S) \times b_{1}^{\alpha}(S)$ matrices over $\mathbb{Z}$ it now follows clearly that $\operatorname{det}\left(i_{+}\right)$equals the top coefficient of $\Delta_{\phi}^{\alpha}$, namely 1 . Similarly $\operatorname{det}\left(i_{-}\right)=1$. This shows that $i_{+}: H_{1}^{\alpha}(S ; \mathbb{Z}[G]) \rightarrow H_{1}^{\alpha}(M ; \mathbb{Z}[G])$ is an isomorphism.

Summarizing, this discussion shows that Conjecture 4.2 follows from the Geometrization Conjecture and the following question in the theory of 3-manifold groups.

Conjecture 8.3. Let $S$ be an incompressible surface in a 3-manifold $N$ and let $M$ be $N$ cut along $S$. Let $i: S \rightarrow M$ be one of the positive and the negative inclusions of $S$ into $M$. If $i: H_{1}^{\alpha}(S ; \mathbb{Z}[G]) \rightarrow H_{1}^{\alpha}(M ; \mathbb{Z}[G])$ is an isomorphism for every homomorphism $\pi_{1}(N) \rightarrow G$, $G$ a finite group, then $i: \pi_{1}(S) \rightarrow \pi_{1}(M)$ is surjective.

Note that it is well-known that the inclusion induced homomorphisms $\pi_{1}(S) \rightarrow \pi_{1}(N)$ and $\pi_{1}(M) \rightarrow \pi_{1}(N)$ are injections. We think that an affirmative answer to the above conjecture will need a strong condition on $\pi_{1}(N)$ (of which $\pi_{1}(M)$ and $\pi_{1}(S)$ are subgroups) such as subgroup separability, which is conjectured to hold for fundamental groups of hyperbolic manifolds (cf. [Th82]).

\section{REFERENCES}

[Ba01] S. Baldridge, Seiberg-Witten invariants of 4-manifolds with free circle actions, Commun. Contemp. Math. 3: 341-353 (2001).

[Ch03] J. Cha, Fibred knots and twisted Alexander invariants, Transactions of the AMS 355: 4187-4200 (2003)

[CG85] J. Cheeger, M. Gromov, Bounds on the von Neumann dimension of $L^{2}$-cohomology and the GaussBonnet theorem for open manifolds, J. Differential Geom. 21, no. 1: 1-34 (1985)

[COT04] T. Cochran, K. Orr, P. Teichner, Structure in the classical knot concordance group, Comment. Math. Helv. 79: 105-123 (2004)

[CF77] R. Crowell, R. Fox, Introduction to knot theory, Graduate Text in Mathematics 57, Springer Verlag (1977)

[Du01] N. Dunfield, Alexander and Thurston norm of fibered 3-manifolds, Pacific J. Math., 200, no. 1: 43-58 (2001)

[Du85] M. J. Dunwoody, An equivariant sphere theorem, Bull. London Math. Soc. 17, no. 5, 437-448 (1985)

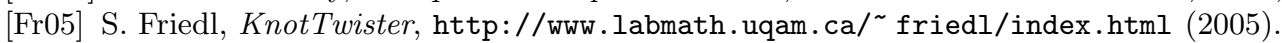

[FK05] S. Friedl, T. Kim, Twisted Alexander norms give lower bounds on the Thurston norm, Trans. Amer. Math. Soc. (to appear)

[FK06] S. Friedl, T. Kim, The Thurston norm, fibered manifolds and twisted Alexander polynomials, Topology 45, 929-953 (2006)

[FV07] S. Friedl, S. Vidussi, Symplectic $\mathbf{S}^{\mathbf{1}} \times \mathbf{N}^{\mathbf{3}}$, surface subgroup separability, and totally degenerate Thurston norm, Preprint (2007), to appear in J. Amer. Math. Soc.

[Ga83] D. Gabai, Foliations and the topology of 3-manifolds, J. Differential Geometry 18, no. 3: 445-503 (1983) 
[Ga87] D. Gabai, Foliations and the topology of 3-manifolds. III, J. Differential Geometry 26, no. 3: 479-536 (1987)

[GKM05] H. Goda, T. Kitano, T. Morifuji, Reidemeister Torsion, Twisted Alexander Polynomial and Fibred Knots, Comment. Math. Helv. 80, no. 1: 51-61 (2005)

[GS99] R. Gompf, A. Stipsicz, 4-manifolds and Kirby calculus, Graduate Studies in Mathematics 20, AMS (1999)

[He87] J. Hempel, Residual finiteness for 3-manifolds, Combinatorial group theory and topology (Alta, Utah, 1984), 379-396, Ann. of Math. Stud., 111, Princeton Univ. Press, Princeton, NJ (1987)

[HT] J. Hoste, M. Thistlethwaite, Knotscape, http://www.math.utk.edu/ morwen/knotscape.html

[JW93] B. Jiang, S. Wang, Twisted topological invariants associated with representations, Topics in knot theory (Erzurum 1992), 211-227, NATO Adv. Sci. Inst. C. Math. Phys. Sci, 399, Kluwer Acad. Publ., Dordrecht (1993)

[KL99] P. Kirk, C. Livingston, Twisted Alexander invariants, Reidemeister torsion and Casson-Gordon invariants, Topology 38, no. 3: 635-661 (1999)

[Kr98] P. Kronheimer, Embedded surfaces and gauge theory in three and four dimensions, Surveys in differential geometry, Vol. III (Cambridge, MA, 1996), 243-298, Int. Press, Boston, MA (1998)

[Kr99] P. Kronheimer, Minimal genus in $S^{1} \times M^{3}$, Invent. Math. 135, no. 1: 45-61 (1999)

[Le67] J. Levine, A method for generating link polynomials, Amer. J. Math. 89 (1967) 69-84.

[L01] X. S. Lin, Representations of knot groups and twisted Alexander polynomials, Acta Math. Sin. (Engl. Ser.) 17, no. 3: 361-380 (2001)

[McC01] J. McCarthy, On the asphericity of a symplectic $M^{3} \times S^{1}$, Proc. Amer. Math. Soc. 129: 257-264 (2001)

[McM02] C. T. McMullen, The Alexander polynomial of a 3-manifold and the Thurston norm on cohomology, Ann. Sci. Ecole Norm. Sup. (4) 35, no. 2: 153-171 (2002)

[MT99] C. T. McMullen, C. H. Taubes, 4-manifolds with inequivalent symplectic forms and 3-manifolds with inequivalent fibrations, Math. Res. Lett. 6: 681-696 (1999)

[MeT96] G. Meng, C. H. Taubes, SW= Milnor torsion, Math. Res. Lett. 3: 661-674 (1996)

[Ro96] D. Robinson, A Course in the Theory of Groups, Graduate Texts in Mathematics 80, Springer Verlag (1996)

[St62] J. Stallings, On fibering certain 3-manifolds, 1962 Topology of 3-manifolds and related topics (Proc. The Univ. of Georgia Institute, 1961) pp. 95-100 Prentice-Hall, Englewood Cliffs, N.J. (1962)

[Sto] A. Stoimenow, http://www.ms.u-tokyo.ac.jp/ stoimeno/ptab/index.html

[Ta98] C. H. Taubes, The geometry of the Seiberg-Witten invariants, Doc. Math. J. DMV Extra Volume ICM II: 493-492 (1998)

[Th76] W. P. Thurston, Some simple examples of symplectic manifolds, Proc. Amer. Math. Soc. 55 (1976), no. $2,467-468$.

[Th82] W. P. Thurston, Three dimensional manifolds, Kleinian groups and hyperbolic geometry, Bull. Amer. Math. Soc. 6 (1982)

[Th86] W. P. Thurston, A norm for the homology of 3-manifolds, Mem. Amer. Math. Soc. 339: 99-130 (1986)

[Tu86] V. Turaev, Reidemeister torsion in knot theory, Russian Math. Surveys 41, no. 1: 119-182 (1986)

[Tu01] V. Turaev, Introduction to combinatorial torsions, Birkhäuser, Basel, (2001)

[Vi99] S. Vidussi, The Alexander norm is smaller than the Thurston norm; a Seiberg-Witten proof, Prepublication Ecole Polytechnique 6 (1999)

[Vi03] S. Vidussi, Norms on the cohomology of a 3-manifold and $S W$ theory, Pacific J. Math. 208, no. 1: 169-186 (2003)

[Wa94] M. Wada, Twisted Alexander polynomial for finitely presentable groups, Topology 33, no. 2: 241-256 (1994) 
Université du QuéBec À Montréal, Montréal, Quèbec

E-mail address: friedl@alumni.brandeis.edu

Department of Mathematics, University of California, Riverside, CA 92521, USA

E-mail address: svidussi@math.ucr.edu 